\title{
Update on the Combined Analysis of Muon Measurements from Nine Air Shower Experiments
}

\section{Dennis Soldin $^{a, *}$ for the EAS-MSU, IceCube, KASCADE-Grande, NEVOD-DECOR, Pierre Auger, SUGAR, Telescope Array, and Yakutsk EAS Array Collaborations}

(a complete list of authors can be found at the end of the proceedings)

\author{
${ }^{a}$ Bartol Research Institute, Dept. of Physics and Astronomy \\ University of Delaware, Newark, DE 19716, USA \\ E-mail: soldin@udel.edu
}

\begin{abstract}
Over the last two decades, various experiments have measured muon densities in extensive air showers over several orders of magnitude in primary energy. While some experiments observed differences in the muon densities between simulated and experimentally measured air showers, others reported no discrepancies.

We will present an update of the meta-analysis of muon measurements from nine air shower experiments, covering shower energies between a few $\mathrm{PeV}$ and tens of $\mathrm{EeV}$ and muon threshold energies from a few $100 \mathrm{MeV}$ to about $10 \mathrm{GeV}$. In order to compare measurements from different experiments, their energy scale was cross-calibrated and the experimental data has been compared using a universal reference scale based on air shower simulations. Above $10 \mathrm{PeV}$, we find a muon excess with respect to simulations for all hadronic interaction models, which is increasing with shower energy. For EPOS-LHC and QGSJet-II.04 the significance of the slope of the increase is analyzed in detail under different assumptions of the individual experimental uncertainties.
\end{abstract}

$37^{\text {th }}$ International Cosmic Ray Conference (ICRC 2021)

July 12 th - 23rd, 2021

Online - Berlin, Germany

\footnotetext{
${ }^{*}$ Presenter
} 


\section{Introduction}

Cosmic rays enter the Earth's atmosphere where they produce Extensive Air Showers (EAS) which can be measured at the ground. Although the energy spectrum of cosmic rays has been measured with high precision over many orders of magnitude, the sources of cosmic rays are still unknown, their acceleration mechanism and mass composition are uncertain, and several features observed in the energy spectrum are not well understood [1,2].

The main challenge lies in the measurements of the muon content in air showers, which have been performed by many experiments over the last 20 years. Various experiments reported discrepancies in the number of muons in simulated and observed air showers, such as the HiRes-MIA [3] and NEVOD-DECOR [4, 5] collaborations, as well as the Pierre Auger Observatory [6, 7] (Auger), Telescope Array [8] (TA), and SUGAR [9]. In contrast, no discrepancies in the average muon densities were observed by EAS-MSU [10], the Yakutsk EAS array [11], and KASCADE-Grande [12]. KASCADE-Grande, however, reported differences in the muon number evolution with the zenith angle with respect to model predictions.

In this article, we present an update of the meta-analysis of global measurements of the lateral muon density by multiple EAS experiments which was previously reported in Refs. [13, 14]. This update includes new data from Auger [15] and its Underground Muon Detectors [16] (UMD), and from the IceCube Neutrino Observatory [17] (IceCube). In addition, data from the AGASA experiment [18] is included for the first time and systematic studies of the energy-dependent trend of the muon discrepancies are discussed in detail. An overview of further measurements of the muon content in EAS is beyond the scope of this work and can be found in Refs. [13, 19].

\section{Measurements of the Muon Lateral Density}

The lateral density of muons measured at the ground depends on various parameters: cosmicray energy, $E$, zenith angle, $\theta$, shower age (vertical depth, $X$, and zenith angle), lateral distance, $r$, from the shower axis, and energy threshold, $E_{\mu, \min }$, of the muon detectors. The parameter space covered by the experiments considered in this meta-analysis is shown in Fig. 1. Due to different experimental conditions and analysis techniques, a direct comparison of the muon measurements is not possible. Instead, to compare different results in a meaningful way, the measurements of each

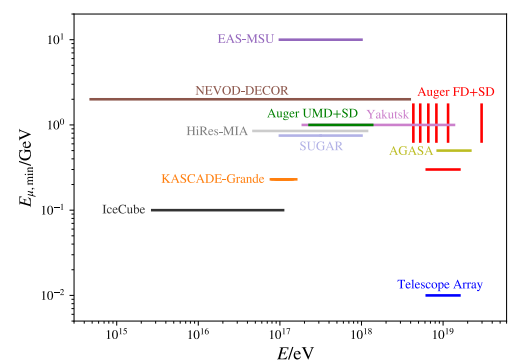

(a)

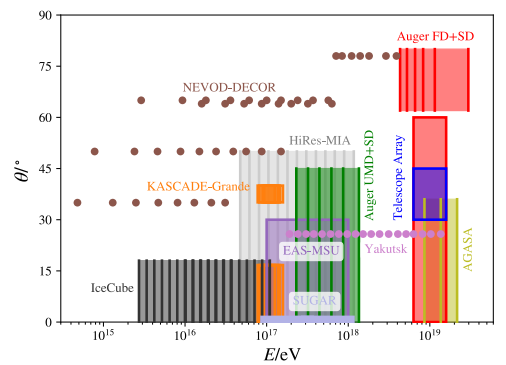

(b)

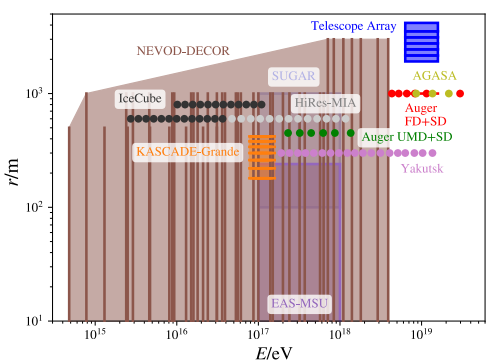

(c)

Figure 1: Phase space of EAS experiments which have reported measurements of the muon density. Points and lines indicate a measurement in a narrow bin of the parameter, while boxes indicate integration over a parameter range. Figure (a) shows the muon energy thresholds, $E_{\mu, \min }$, (b) the zenith angle range, $\theta$, and (c) the lateral distances, $r$, of the muon density measurements, as a function of the EAS energy, $E$. 


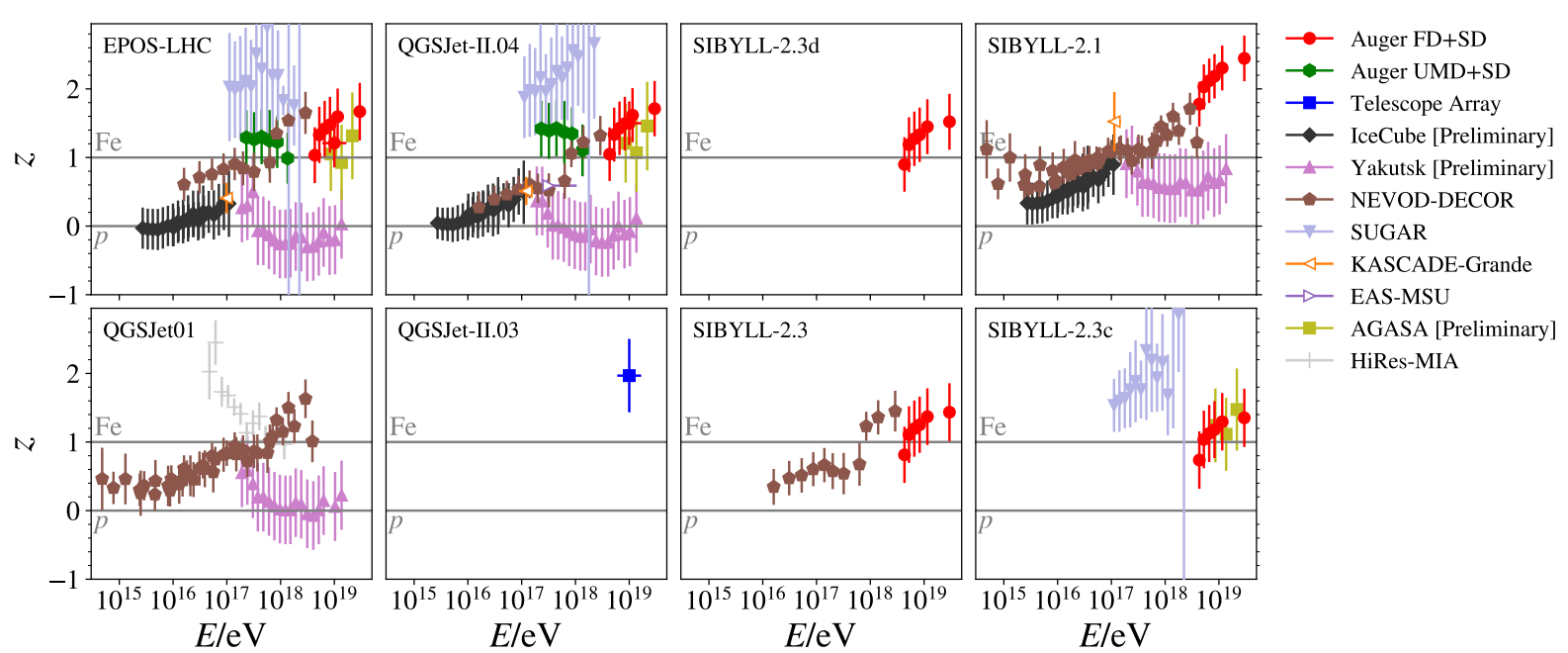

Figure 2: Muon density measurements converted to the z-scale, as defined in Eq. (1), for different hadronic interaction models. When corresponding simulations are missing for an experiment, no points can be shown. Error bars show statistical and systematic uncertainties added in quadrature.

experiment have to be compared to EAS simulations in the same observation conditions in terms of a data/MC ratio. Therefore, the measurements of the muon density from different experiments are converted to the $z$-scale,

$$
z=\frac{\ln \left\langle N_{\mu}^{\mathrm{det}}\right\rangle-\ln \left\langle N_{\mu, \mathrm{p}}^{\mathrm{det}}\right\rangle}{\ln \left\langle N_{\mu, \mathrm{Fe}}^{\mathrm{det}}\right\rangle-\ln \left\langle N_{\mu, \mathrm{p}}^{\mathrm{det}}\right\rangle},
$$

where $\left\langle N_{\mu}^{\mathrm{det}}\right\rangle$ is the average muon density estimate as seen in the detector, while $\left\langle N_{\mu, \mathrm{p}}^{\mathrm{det}}\right\rangle$ and $\left\langle N_{\mu, \mathrm{Fe}}^{\mathrm{det}}\right\rangle$ are the simulated average muon densities for proton and iron showers after a full detector simulation. The $\mathrm{z}$-scale is constructed such that an observation of the muon density of $z=0$ is consistent with a simulated proton shower and $z=1$ for a simulated iron shower. A dedicated discussion on the properties and calculation of the z-scale and its uncertainties can be found in Ref. [18].

The z-values obtained by nine air shower experiments [4-12, 15-18] are shown in Fig. 2. Depending on the experiment, the distributions are given for the post-LHC hadronic interaction models EPOS-LHC [20], QGSJet-II.04 [21], and Sibyll 2.3(c/d) [22, 23], and for the pre-LHC models QGSJet01 and QGSJet-II.03 [24], and Sibyll 2.1 [25]. For all models the data lies between the expectations for proton and iron showers up to energies of about $10^{17} \mathrm{eV}$. However, at higher energies all data except Yakutsk suggest an unphysical mass composition heavier than iron.

\subsection{Energy Scale Offsets and Cross-Calibration}

According to the Matthews-Heitler model [26] the number of muons in EAS, $N_{\mu}$, depends on the energy, $E$, and mass, $A$, of the initial cosmic ray as

$$
N_{\mu}=A^{1-\beta} \cdot\left(E / \xi_{C}\right)^{\beta}
$$

with power-law index $\beta \simeq 0.9$ and energy constant, $\xi_{C}$. This causes two experiments with an energy-scale offset of $20 \%$, for example, to have an $18 \%$ offset in the data/MC ratios because measurements are compared to EAS simulated at different apparent energies. To compare muon measurements between experiments, energy-scale offsets therefore need to be taken into account. 
Assuming that the cosmic ray flux is a universal reference and that all deviations in measured fluxes between different experiments arise from energy scale offsets, a relative scale $E_{\text {data }} / E_{\text {ref }}$ can be determined for each experiment such that the all-particle fluxes match [27]. The relative energy-scale shift between Auger and TA has been found to be $10.4 \%$ [28] and the reference energy scale, $E_{\text {ref }}$, used in this work is placed between the two experiments, as shown in Fig. 3. The scaling factors for the other experiments are obtained from the Global Spline Fit (GSF) flux model [27] which also uses cross-calibration internally, with a reference energy scale $E_{\text {ref, GSF }} / E_{\text {ref }}=0.948 / 0.880 \simeq 1.08$. Using the adjustment factors from Fig. 3, the z-values are energy cross-calibrated as described in detail in Ref. [13] and the individual uncertainties of each experiment are adjusted by removing the contribution from the energy-scale. However, the reference energy-scale,

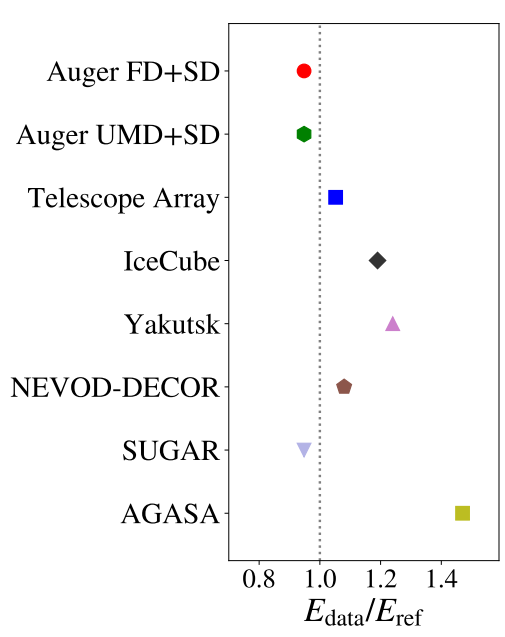

Figure 3: Energy-scale adjustment factors obtained from the crosscalibration described in Ref. [27]. after cross-calibration, has a remaining uncertainty of at least $10 \%$, causing potential shifts of the z-values by about \pm 0.25 . No cross-calibration factor can be given for KASCADE-Grande because the cosmic ray flux is computed using a different energy estimator to which this method can not be applied [13]. For EAS-MSU, no all-particle flux is available for cross-calibration.

The resulting z-values, after applying the energy-scale cross-calibration, are shown in Fig. 4, where a remarkably consistent picture is obtained. The measurements are in agreement with simulations based on the post-LHC hadronic interaction models, EPOS-LHC and QGSJetII.04, up to about a few $10^{16} \mathrm{eV}$, within the expectation from measurements of the maximum shower depth, $X_{\max }$, and uncertainties. However, at higher energies, an increasing muon excess with respect to simulations is observed for all models, suggesting a mass composition heavier than iron.

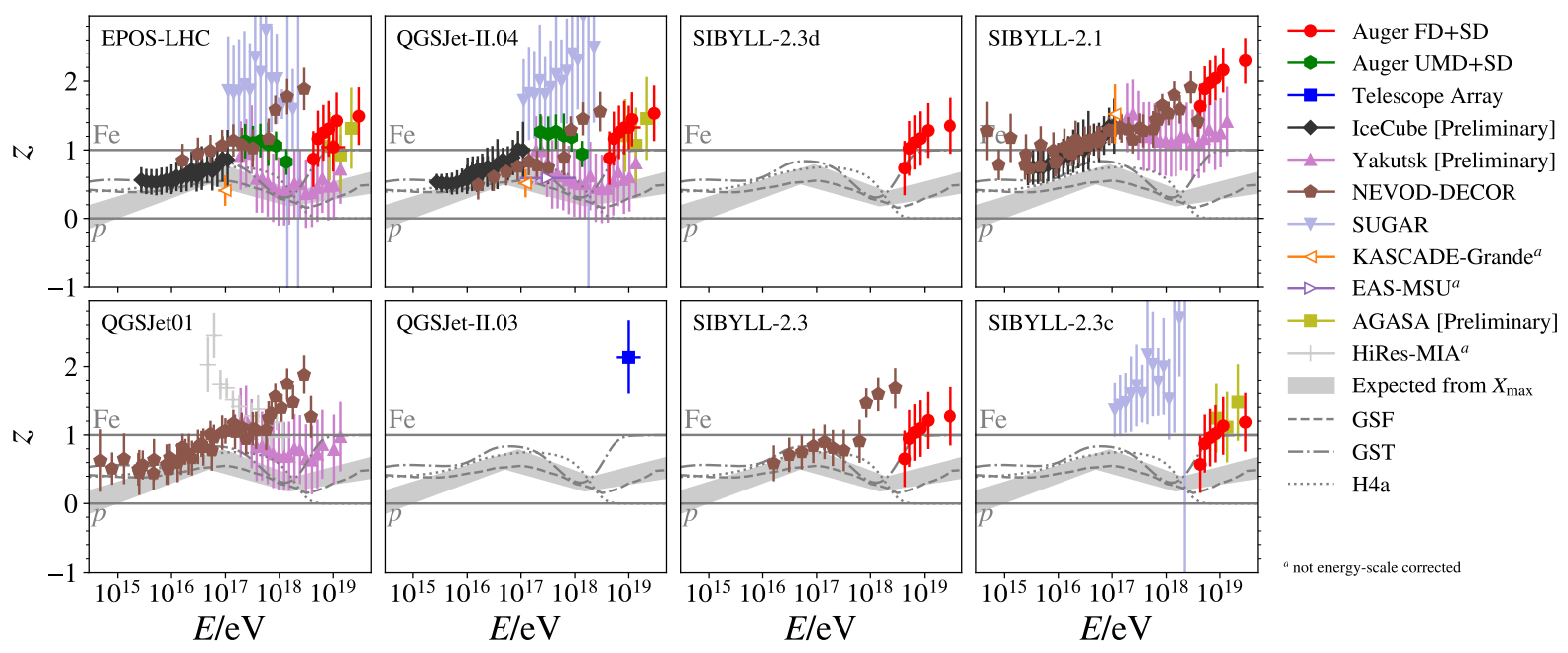

Figure 4: Combined data from Fig. 2 after applying energy-scale cross-calibration, as described in the text. The data of KASCADE-Grande and EAS-MSU cannot be cross-calibrated and are only included for comparison. Shown for comparison are z-values expected for a mixed composition from optical measurements $\left(X_{\max }\right)$, based on an update of Ref. [1], and from the flux models GSF [27], GST [29], and H4a [30]. 

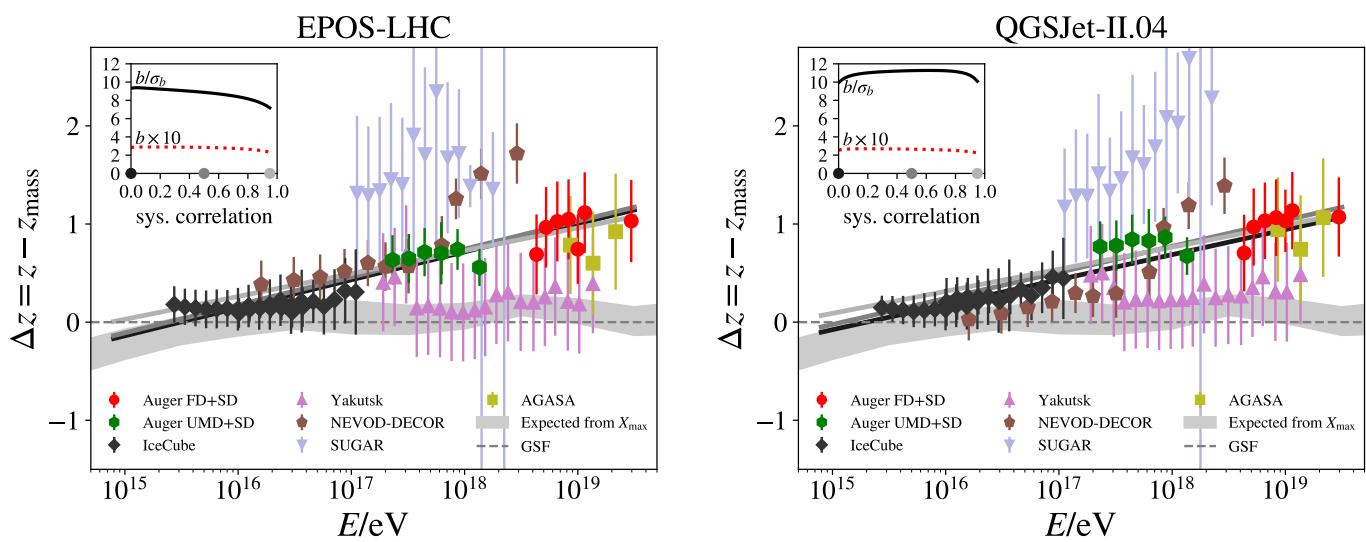

Figure 5: Linear fits to the $\Delta z=z-z_{\text {mass }}$ distributions, as described in Eq. (3). Shown in the inset are the slope, $b$, and its deviation from zero in standard deviations for an assumed correlation of the point-wise uncertainties within each experiment. Examples of the fits are shown for a correlation of 0.0, 0.5, and 0.95.

\subsection{Energy-Dependent Trend}

In order to systematically quantify the energy-dependent trend observed in the muon measurements shown in Fig. 4, the mass composition dependence expected from Eq. (2) needs to be taken into account. If the measured z-values follow $z_{\text {mass }}$ as expectated from $X_{\max }$ measurements, the model describes the muon density at the ground consistently. Subtracting $z_{\text {mass }}$ is thus expected to remove the effect of the changing mass composition. The resulting $\Delta z=z-z_{\text {mass }}$ distributions are shown in Fig. 5 for EPOS-LHC and QGSJet-II.04 with $z_{\text {mass }}$ determined from the GSF flux model [27]. A simple linear function of the form

$$
\Delta z_{\mathrm{fit}}=a+b \cdot \log _{10}\left(E / 10^{16} \mathrm{eV}\right)
$$

is fit to the data, where $a$ and $b$ are free parameters. To account for (the unknown) correlated uncertainties in the experimental data, the least-squares method described in Ref. [13] is used. It assumes a correlation factor, $\alpha$, between data points belonging to the same data set. The fit is repeated for values of $\alpha$ between 0 and 0.95 . To adjust for over/under-estimated uncertainties, the raw result, $\sigma_{b}^{\text {raw }}$, is re-scaled with the $\chi^{2}$ value and the degrees of freedom, $n_{\mathrm{dof}}$, of the fit as $\sigma_{b}=\sigma_{b}^{\text {raw }} \cdot \sqrt{\chi^{2} / n_{\text {dof }}}$, as described in Ref. [13]. For EPOS-LHC, the resulting slope ranges from
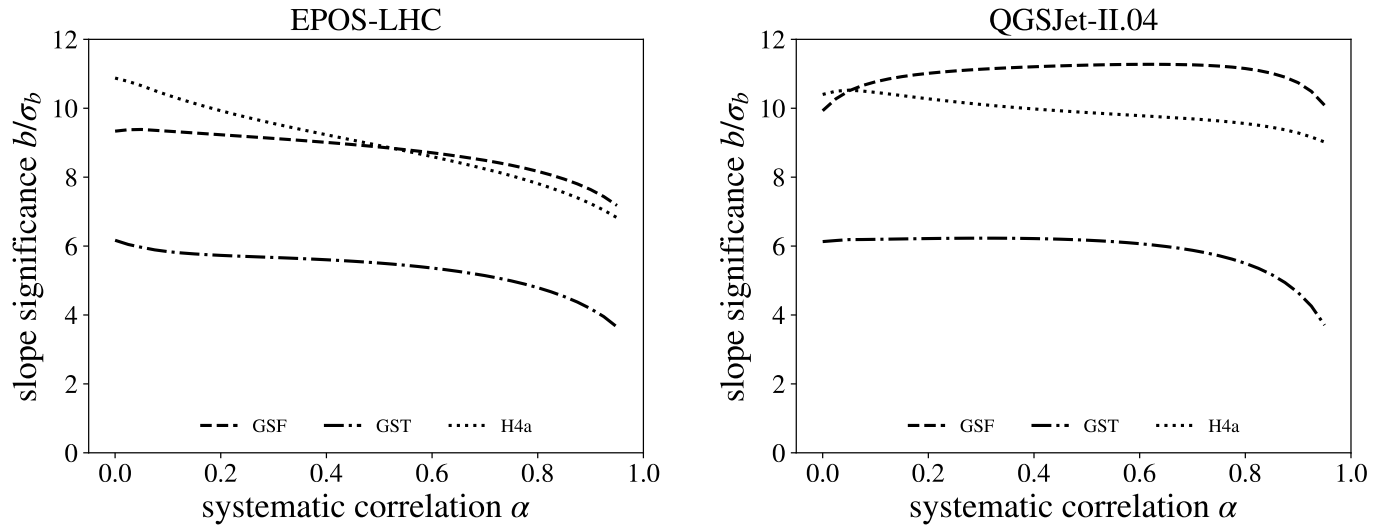

Figure 6: Significance of the deviation of the slope, $b$, in Eq. (3) from zero as a function of an assumed correlation, $\alpha$, of the uncertainties within each experiment. The significances are shown for $\Delta z$ with $z_{\text {mass }}$ determined from the GSF, GST, and H4a models. 


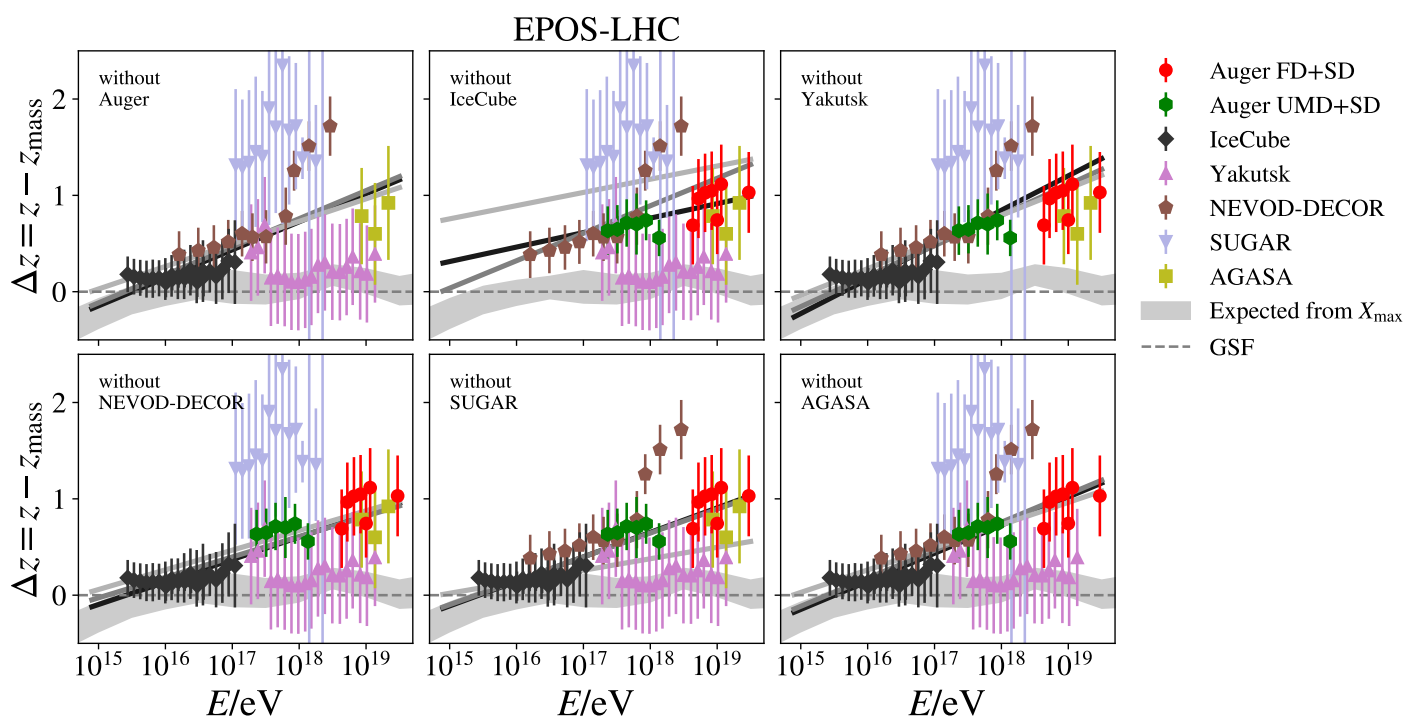

Figure 7: Linear fits to the data points as described in Eq. (3) with individual experiments excluded from the data. Examples of the fits are shown for systematic correlations of 0.0, 0.5, and 0.95.

$b=0.23 \pm 0.03$ up to $b=0.29 \pm 0.03$ for $\alpha=0.95$ and $\alpha=0.0$, respectively. For QGSJet-II.04, it ranges from $b=0.22 \pm 0.02$ up to $b=0.25 \pm 0.03$ for $\alpha=0.95$ and $\alpha=0.0$. The significances of the deviations from $b=0.0$ are around $8 \sigma$ for EPOS-LHC and above $10 \sigma$ for QGSJet-II.04.

To study the influence of the underlying mass assumption, the fits in $\Delta z=z-z_{\text {mass }}$ are repeated with $z_{\text {mass }}$ obtained from the GST [29] and H4a [30] flux models which are also based on fits to experimental data. The resulting significances for correlations between 0.0 and 0.95 are shown in Fig. 6. While the significances based on the H4a model increase, the GST model always yields smaller significances. However, as shown in Fig. 4, the GST model predicts a heavier cosmic ray mass composition which is in strong tension with measurements of $X_{\max }$ over the vast majority of the energy range considered here. This confirms the choice of the GSF model which shows the best overall agreement with optical measurements of the mass composition. In addition, the effect of the choice of the reference energy scale, $E_{\text {ref }}$ in Fig. 3, was also studied and found to be negligible.

\subsection{N-1 Tests}

To study the contribution from each individual experiment to the significances of the fit slopes to the combined data, systematic $N-1$ tests are performed where data from one experiment at a time is excluded from the fit. The resulting fits for EPOS-LHC are shown in Fig. 7 and the corresponding significances for the models EPOS-LHC and QGSJet-II.04 are depicted in Fig. 8 for systematic correlations $\alpha$ between 0.0 and 0.95 . The significances of the fit slopes remain above $5 \sigma$ when excluding most experiments. However, lower significances can be observed for EPOS-LHC, for extreme correlations, when removing data from IceCube, and to some extent when removing data from SUGAR. In contrast, excluding the measurements by Yakutsk causes an increase of the significances, in particular towards very small correlations. This indicates that measurements from Yakutsk, which are in strong tension with other data in the same energy region, have a stronger influence on the fit result if data from IceCube is removed. These effects are more pronounced for EPOS-LHC compared to QGSJet-II.04. This is due to a smaller scatter of the data for QGSJet-II.04, which causes smaller uncertainties in the slope, $b$, of the fit through the $\chi^{2} / n_{\text {dof }}$ re-scaling. 

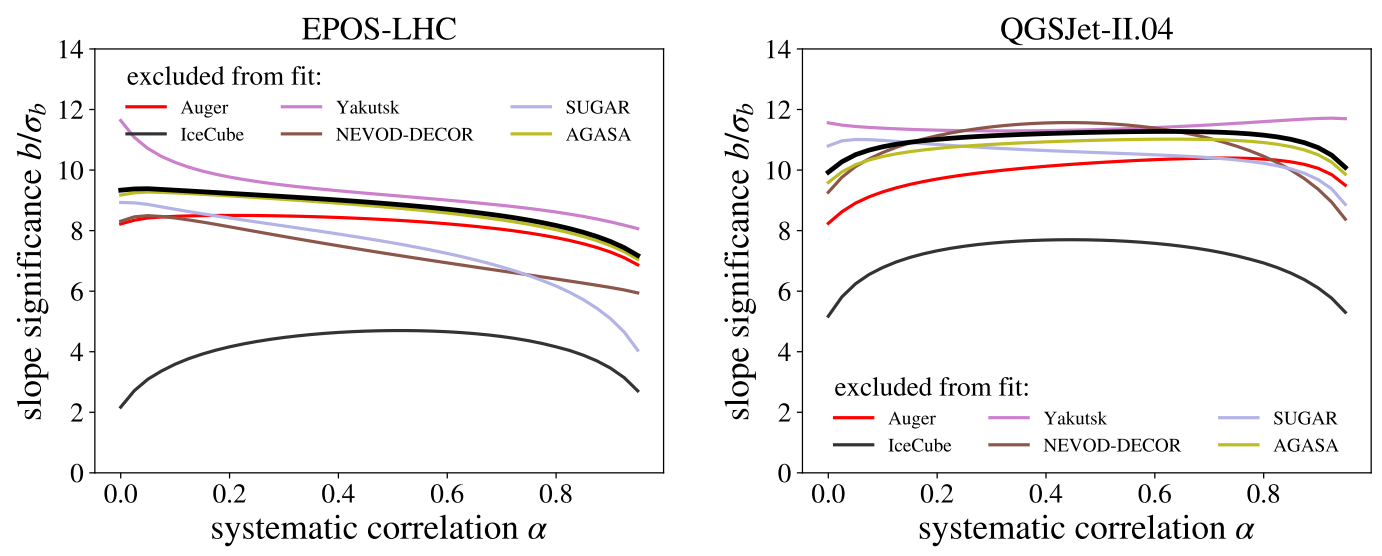

Figure 8: Significance of the deviation of the slope, $b$, in Eq. (3) from zero as a function of an assumed systematic correlation of the uncertainties within each experiment, $\alpha$. Black lines show the result from Fig. 5 and colored lines the results where individual experiments are excluded from the fit (see text for details).

\section{Conclusions}

An update of the meta-analysis of muon measurements in EAS with energies from $\mathrm{PeV}$ up to tens of EeV was presented and a remarkably consistent picture is obtained after cross-calibrating the energy scales of the different experiments. The measurements agree with simulations based on the models EPOS-LHC and QGSJet-II.04, within uncertainties, up to energies of a few $10^{16} \mathrm{eV}$, assuming the GSF flux model. At higher energies, an increasing excess with respect to model predictions is observed. The slope of this increase is between $b=0.23$ and $b=0.29$ for EPOSLHC and between $b=0.22$ and $b=0.25$ for QGSJet-II.04, with a significance of the deviations from $b=0.0$ of around $8 \sigma$ and $10 \sigma$, respectively. A small change of the fits compared to the previous meta-analysis $[13,14]$ is observed which arises mainly from the updates of the experimental data. Studies of other dependencies, for example the minimum energy of muons at production for each experiment, as described in Ref. [14], have yet been inconclusive due to the limited experimental data. It has been shown that the mass composition model is important for the mass subtraction in $\Delta z=z-z_{\text {mass }}$ and should be derived from optical measurements with the least amount of assumptions. The GSF model satisfies this requirement at a certain level, however, the possibility to use data from $X_{\max }$ measurements directly is currently under investigation.

When removing individual data from this meta-analysis, for extreme assumptions of the correlation of uncertainties, the significances for a non-zero slope can decrease to approximately $3 \sigma$ for EPOS-LHC and around $5 \sigma$ for QGSJet-II.04. This decrease is mainly due to the data from Yakutsk, which becomes more important when data from IceCube (or to some extent SUGAR) are removed and is in strong tension with other muon measurements. To understand these tensions, further studies of the treatment of systematic uncertainties and relative biases of the individual experiments are necessary. Moreover, additional measurements with high precision are needed, in particular at energies above $10^{17} \mathrm{eV}$. Ongoing EAS detector upgrades and improved analysis methods are expected to reduce the uncertainties of the experimental results and increase the parameter space. This will improve the data accuracy and help to investigate other dependencies of the deviations between simulations and data in future extensions of this meta-analysis. 


\section{References}

[1] K.-H. Kampert and M. Unger Astropart. Phys. 35 (2012) 660-678.

[2] J. Becker Tjus and L. Merten Phys. Rept. 872 (2020) 1-98.

[3] HiRes-MIA Collaboration, T. Abu-Zayyad et al. Phys. Rev. Lett. 84 (2000) 4276-4279.

[4] NEVOD-DECOR Collaboration, A. G. Bogdanov et al. Phys. Atom. Nucl. 73 (2010) .

[5] NEVOD-DECOR Collaboration, A. G. Bogdanov et al. Astropart. Phys. 98 (2018) 13-20.

[6] Pierre Auger Collaboration, A. Aab et al. Phys. Rev. D 91 no. 3, (2015) 032003.

[7] Pierre Auger Collaboration, A. Aab et al. Phys. Rev. Lett. 117 no. 19, (2016) 192001.

[8] Telescope Array Collaboration, R. U. Abbasi et al. Phys. Rev. D 98 no. 2, (2018) 022002.

[9] SUGAR Collaboration, J. A. Bellido et al. Phys. Rev. D 98 no. 2, (2018) 023014.

[10] EAS-MSU Collaboration, Y. A. Fomin et al. Astropart. Phys. 92 (2017) 1-6.

[11] Yakutsk Collaboration, A. Glushkov, M. Pravdin, and A. Sabourov Priv. Comm. (2018) .

[12] KASCADE-Grande Collaboration, W. D. Apel et al. Astropart. Phys. 95 (2017) 25-43.

[13] EAS-MSU, IceCube, KASCADE Grande, NEVOD-DECOR, Pierre Auger, SUGAR, Telescope Array, Yakutsk EAS Array Collaboration, H. P. Dembinski et al. EPJ Web Conf. 210 (2019) 02004.

[14] EAS-MSU, IceCube, KASCADE Grande, NEVOD-DECOR, Pierre Auger, SUGAR, Telescope Array, Yakutsk EAS Array Collaboration, L. Cazon PoS ICRC2019 (2020) 214.

[15] Pierre Auger Collaboration, A. Aab et al. Phys. Rev. Lett. 126 no. 15, (2021) 152002.

[16] Pierre Auger Collaboration, A. Aab et al. Eur. Phys. J. C 80 no. 8, (2020) 751.

[17] IceCube Collaboration, D. Soldin PoS ICRC2021 (2021) 342.

[18] F. Gesualdi et al. PoS ICRC2021 (2021) 473.

[19] J. Albrecht et al. submitted to Astrophysics and Space Science (2021) .

[20] T. Pierog et al. Phys. Rev. C 92 no. 3, (2015) 034906.

[21] S. Ostapchenko EPJ Web Conf. 52 (2013) 02001.

[22] F. Riehn et al. PoS ICRC2017 (2018) 301.

[23] F. Riehn et al. Phys. Rev. D 102 no. 6, (2020) 063002.

[24] N. Kalmykov and S. Ostapchenko Phys. Atom. Nucl. 56 no. 346, (1993) .

[25] E.-J. Ahn et al. Phys. Rev. D 80 (2009) 094003.

[26] J. Matthews Astropart. Phys. 22 (2005) 387-397.

[27] H. P. Dembinski et al. PoS ICRC2017 (2018) 533.

[28] Pierre Auger, Telescope Array Collaboration, O. Deligny PoS ICRC2019 (2020) 234.

[29] T. K. Gaisser, T. Stanev, and S. Tilav Front. Phys.(Beijing) 8 (2013) 748-758.

[30] T. K. Gaisser Astropart. Phys. 35 (2012) 801-806. 


\section{Full Author List:}

\section{EAS-MSU Collaboration:}

Yu. A. Fomin ${ }^{1}$, N. N. Kalmykov ${ }^{1}$, I. S. Karpikov ${ }^{2}$, G. V. Kulikov ${ }^{1}$, M. Yu. Kuznetsov ${ }^{2,3}$, G. I. Rubtsov ${ }^{2}$, V. P. Sulakov ${ }^{1}$, S. V. Troitsky ${ }^{2}$

${ }^{1}$ D.V. Skobeltsyn Institute of Nuclear Physics, M.V. Lomonosov Moscow State University, Moscow 119991, Russia

2 Institute for Nuclear Research of the Russian Academy of Sciences, Moscow, Russia

${ }^{3}$ Service de Physique Théorique, Université Libre de Bruxelles, Brussels, Belgium

\section{IceCube Collaboration:}

R. Abbasi ${ }^{17}$, M. Ackermann ${ }^{59}$, J. Adams ${ }^{18}$, J. A. Aguilar ${ }^{12}$, M. Ahlers ${ }^{22}$, M. Ahrens ${ }^{50}$, C. Alispach ${ }^{28}$, A. A. Alves Jr. ${ }^{31}$, N. M. $\mathrm{Amin}^{42}$, R. An ${ }^{14}$, K. Andeen ${ }^{40}$, T. Anderson ${ }^{56}$, G. Anton ${ }^{26}$, C. Argüelles ${ }^{14}$, Y. Ashida ${ }^{38}$, S. Axani ${ }^{15}$, X. Bai ${ }^{46}$, A. Balagopal V. ${ }^{38}$, A. Barbano ${ }^{28}$, S. W. Barwick ${ }^{30}$, B. Bastian ${ }^{59}$, V. Basu ${ }^{38}$, S. Baur ${ }^{12}$, R. Bay ${ }^{8}$, J. J. Beatty ${ }^{20}, 21$, K.-H. Becker ${ }^{58}$, J. Becker Tjus ${ }^{11}$, C. Bellenghi $^{27}$, S. BenZvi ${ }^{48}$, D. Berley ${ }^{19}$, E. Bernardini ${ }^{59,60}$, D. Z. Besson ${ }^{34,61}$, G. Binder ${ }^{8}$,, D. Bindig ${ }^{58}$, E. Blaufuss ${ }^{19}$, S. Blot ${ }^{59}$, M. Boddenberg ${ }^{1}$, F. Bontempo ${ }^{31}$, J. Borowka ${ }^{1}$, S. Böser ${ }^{39}$, O. Botner ${ }^{57}$, J. Böttcher ${ }^{1}$, E. Bourbeau ${ }^{22}$, F. Bradascio ${ }^{59}$, J. Braun ${ }^{38}$, S. Bron $^{28}$, J. Brostean-Kaiser ${ }^{59}$, S. Browne ${ }^{32}$, A. Burgman ${ }^{57}$, R. T. Burley ${ }^{2}$, R. S. Busse ${ }^{41}$, M. A. Campana ${ }^{45}$, E. G. Carnie-Bronca ${ }^{2}$, C. Chen ${ }^{6}$, D. Chirkin ${ }^{38}$, K. Choi ${ }^{52}$, B. A. Clark ${ }^{24}$, K. Clark ${ }^{33}$, L. Classen ${ }^{41}$, A. Coleman ${ }^{42}$, G. H. Collin ${ }^{15}$, J. M. Conrad ${ }^{15}$, P. Coppin $^{13}$, P. Correa ${ }^{13}$, D. F. Cowen ${ }^{55}, 56$, R. Cross ${ }^{48}$, C. Dappen ${ }^{1}$, P. Dave ${ }^{6}$, C. De Clercq ${ }^{13}$, J. J. DeLaunay ${ }^{56}$, H. Dembinski $^{42}, \mathrm{~K}^{2}$ Deoskar $^{50}$, S. De Ridder ${ }^{29}$, A. Desai ${ }^{38}$, P. Desiati ${ }^{38}$, K. D. de Vries ${ }^{13}$, G. de Wasseige ${ }^{13}$, M. de With ${ }^{10}$, T. DeYoung ${ }^{24}$, S. Dharani ${ }^{1}$, A. Diaz ${ }^{15}$, J. C. Díaz-Vélez ${ }^{38}$, M. Dittmer ${ }^{41}$, H. Dujmovic ${ }^{31}$, M. Dunkman ${ }^{56}$, M. A. DuVernois ${ }^{38}$, E. Dvorak ${ }^{46}$, T. Ehrhardt ${ }^{39}$, P. Eller $^{27}$, R. Engel ${ }^{31,} 32$, H. Erpenbeck ${ }^{1}$, J. Evans ${ }^{19}$, P. A. Evenson ${ }^{42}$, K. L. Fan ${ }^{19}$, A. R. Fazely ${ }^{7}$, S. Fiedlschuster ${ }^{26}$, A. T. Fienberg ${ }^{56}$, K. Filimonov ${ }^{8}$, C. Finley ${ }^{50}$, L. Fischer ${ }^{59}$, D. Fox ${ }^{55}$, A. Franckowiak ${ }^{11,59}$, E. Friedman ${ }^{19}$, A. Fritz ${ }^{39}$, P. Fürst ${ }^{1}$, T. K. Gaisser ${ }^{42}$, J. Gallagher $^{37}$, E. Ganster ${ }^{1}$, A. Garcia ${ }^{14}$, S. Garrappa ${ }^{59}$, L. Gerhardt ${ }^{9}$, A. Ghadimi ${ }^{54}$, C. Glaser ${ }^{57}$, T. Glauch ${ }^{27}$, T. Glüsenkamp ${ }^{26}$, A. Goldschmidt $^{9}$, J. G. Gonzalez ${ }^{42}$, S. Goswami ${ }^{54}$, D. Grant ${ }^{24}$, T. Grégoire ${ }^{56}$, S. Griswold ${ }^{48}$, M. Gündüz ${ }^{11}$, C. Günther ${ }^{1}$, C. Haack ${ }^{27}$,

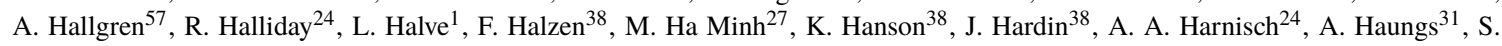

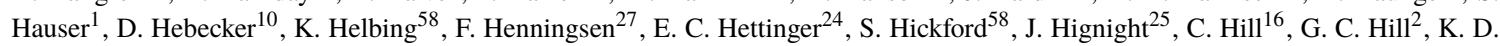

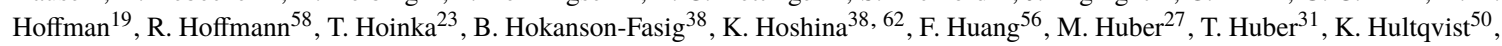
M. Hünnefeld ${ }^{23}$, R. Hussain ${ }^{38}$, S. In ${ }^{52}$, N. Iovine ${ }^{12}$, A. Ishihara ${ }^{16}$, M. Jansson ${ }^{50}$, G. S. Japaridze ${ }^{5}$, M. Jeong ${ }^{52}$, B. J. P. Jones ${ }^{4}$, D. Kang ${ }^{31}$, W. Kang ${ }^{52}$, X. Kang ${ }^{45}$, A. Kappes ${ }^{41}$, D. Kappesser ${ }^{39}$, T. Karg ${ }^{59}$, M. Karl ${ }^{27}$, A. Karle ${ }^{38}$, U. Katz ${ }^{26}$, M. Kauer ${ }^{38}$, M. Kellermann ${ }^{1}$, J. L. Kelley $^{38}$, A. Kheirandish ${ }^{56}$, K. Kin ${ }^{16}$, T. Kintscher ${ }^{59}$, J. Kiryluk ${ }^{51}$, S. R. Klein ${ }^{8,9}$, R. Koirala ${ }^{42}$, H. Kolanoski ${ }^{10}$, T. Kontrimas ${ }^{27}$, L. $\mathrm{Köpke}^{39}$, C. Kopper ${ }^{24}$, S. Kopper ${ }^{54}$, D. J. Koskinen ${ }^{22}$, P. Koundal ${ }^{31}$, M. Kovacevich ${ }^{45}$, M. Kowalski ${ }^{10,59}$, T. Kozynets ${ }^{22}$, E. Kun ${ }^{11}$,

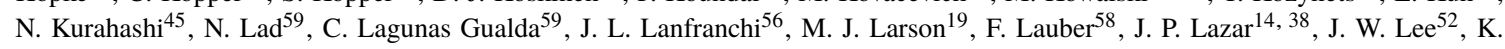
Leonard $^{38}$, A. Leszczyńska ${ }^{32}$, Y. Li ${ }^{56}$, M. Lincetto ${ }^{11}$, Q. R. Liu ${ }^{38}$, M. Liubarska ${ }^{25}$, E. Lohfink ${ }^{39}$, C. J. Lozano Mariscal ${ }^{41}$, L. Lu ${ }^{38}$,

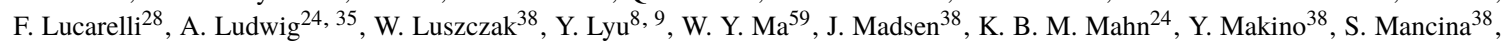
I. C. Mariş ${ }^{12}$, R. Maruyama ${ }^{43}$, K. Mase ${ }^{16}$, T. McElroy ${ }^{25}$, F. McNally ${ }^{36}$, J. V. Mead ${ }^{22}$, K. Meagher $^{38}$, A. Medina ${ }^{21}$, M. Meier ${ }^{16}$, S. Meighen-Berger $^{27}$, J. Micallef ${ }^{24}$, D. Mockler ${ }^{12}$, T. Montaruli ${ }^{28}$, R. W. Moore ${ }^{25}$, R. Morse ${ }^{38}$, M. Moulai $^{15}$, R. Naab ${ }^{59}$, R. Nagai ${ }^{16}$, U. Naumann $^{58}$, J. Necker ${ }^{59}$, L. V. Nguyê̂n ${ }^{24}$, H. Niederhausen ${ }^{27}$, M. U. Nisa ${ }^{24}$, S. C. Nowicki ${ }^{24}$, D. R. Nygren ${ }^{9}$, A. Obertacke Pollmann ${ }^{58}$, M. Oehler ${ }^{31}$, A. Olivas ${ }^{19}$, E. O'Sullivan ${ }^{57}$, H. Pandya ${ }^{42}$, D. V. Pankova ${ }^{56}$, N. Park ${ }^{33}$, G. K. Parker ${ }^{4}$, E. N. Paudel ${ }^{42}$, L. Paul ${ }^{40}$, C. Pérez de los $\operatorname{Heros}^{57}$, L. Peters ${ }^{1}$, J. Peterson ${ }^{38}$, S. Philippen ${ }^{1}$, D. Pieloth ${ }^{23}$, S. Pieper ${ }^{58}$, M. Pittermann ${ }^{32}$, A. Pizzuto ${ }^{38}$, M. Plum ${ }^{40}$, Y. Popovych $^{39}$, A. Porcelli ${ }^{29}$, M. Prado Rodriguez ${ }^{38}$, P. B. Price ${ }^{8}$, B. Pries ${ }^{24}$, G. T. Przybylski ${ }^{9}$, C. Raab ${ }^{12}$, A. Raissi ${ }^{18}$, M. Rameez $^{22}$, K. Rawlins $^{3}$, I. C. Rea ${ }^{27}$, A. Rehman ${ }^{42}$, P. Reichherzer ${ }^{11}$, R. Reimann ${ }^{1}$, G. Renzi ${ }^{12}$, E. Resconi ${ }^{27}$, S. Reusch ${ }^{59}$, W. Rhode ${ }^{23}$, M. Richman ${ }^{45}$, B. Riedel ${ }^{38}$, E. J. Roberts ${ }^{2}$, S. Robertson ${ }^{8,9}$, G. Roellinghoff ${ }^{52}$, M. Rongen ${ }^{39}$, C. Rott ${ }^{49}, 52$, T. Ruhe ${ }^{23}$, D. Ryckbosch ${ }^{29}$, D. Rysewyk $\mathrm{Cantu}^{24}$, I. Safa ${ }^{14,38}$, J. Saffer ${ }^{32}$, S. E. Sanchez Herrera ${ }^{24}$, A. Sandrock ${ }^{23}$, J. Sandroos ${ }^{39}$, M. Santander ${ }^{54}$, S. Sarkar ${ }^{44}$, S. Sarkar ${ }^{25}$, K. Satalecka $^{59}$, M. Scharf ${ }^{1}$, M. Schaufel ${ }^{1}$, H. Schieler ${ }^{31}$, S. Schindler ${ }^{26}$, P. Schlunder ${ }^{23}$, T. Schmidt ${ }^{19}$, A. Schneider ${ }^{38}$, J. Schneider ${ }^{26}$, F. G. Schröder ${ }^{31,42}$, L. Schumacher ${ }^{27}$, G. Schwefer ${ }^{1}$, S. Sclafani ${ }^{45}$, D. Seckel ${ }^{42}$, S. Seunarine ${ }^{47}$, A. Sharma ${ }^{57}$, S. Shefali ${ }^{32}$, M. Silva ${ }^{38}$, B. Skrzypek ${ }^{14}$, B. Smithers ${ }^{4}$, R. Snihur ${ }^{38}$, J. Soedingrekso ${ }^{23}$, D. Soldin ${ }^{42}$, C. Spannfellner ${ }^{27}$, G. M. Spiczak ${ }^{47}$, C. Spiering ${ }^{59,}$, 61, J. Stachurska $^{59}$, M. Stamatikos ${ }^{21}$, T. Stanev ${ }^{42}$, R. Stein ${ }^{59}$, J. Stettner ${ }^{1}$, A. Steuer ${ }^{39}$, T. Stezelberger ${ }^{9}$, T. Stürwald ${ }^{58}$, T. Stuttard ${ }^{22}$, G. W. Sullivan $^{19}$, I. Taboada ${ }^{6}$, F. Tenholt ${ }^{11}$, S. Ter-Antonyan ${ }^{7}$, S. Tilav ${ }^{42}$, F. Tischbein ${ }^{1}$, K. Tollefson $^{24}$, L. Tomankova ${ }^{11}$, C. Tönnis ${ }^{53}$, S.

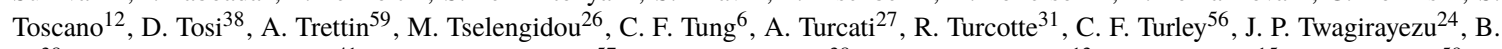
$\mathrm{Ty}^{38}$, M. A. Unland Elorrieta ${ }^{41}$, N. Valtonen-Mattila ${ }^{57}$, J. Vandenbroucke ${ }^{38}$, N. van Eijndhoven ${ }^{13}$, D. Vannerom ${ }^{15}$, J. van Santen $^{59}$, S. Verpoest $^{29}$, M. Vraeghe ${ }^{29}$, C. Walck ${ }^{50}$, T. B. Watson ${ }^{4}$, C. Weaver ${ }^{24}$, P. Weigel ${ }^{15}$, A. Weindl ${ }^{31}$, M. J. Weiss ${ }^{56}$, J. Weldert ${ }^{39}$, C. Wendt $^{38}$, J. Werthebach ${ }^{23}$, M. Weyrauch ${ }^{32}$, N. Whitehorn ${ }^{24}, 35$, C. H. Wiebusch ${ }^{1}$, D. R. Williams ${ }^{54}$, M. Wolf ${ }^{27}$, K. Woschnagg ${ }^{8}$, G. Wrede ${ }^{26}$, J. Wulff ${ }^{11}$, X. W. Xu ${ }^{7}$, Y. Xu ${ }^{51}$, J. P. Yanez ${ }^{25}$, S. Yoshida ${ }^{16}$, S. Yu ${ }^{24}$, T. Yuan ${ }^{38}$, Z. Zhang ${ }^{51}$

\footnotetext{
${ }^{1}$ III. Physikalisches Institut, RWTH Aachen University, D-52056 Aachen, Germany

${ }^{2}$ Department of Physics, University of Adelaide, Adelaide, 5005, Australia

${ }^{3}$ Dept. of Physics and Astronomy, University of Alaska Anchorage, 3211 Providence Dr., Anchorage, AK 99508, USA

${ }^{4}$ Dept. of Physics, University of Texas at Arlington, 502 Yates St., Science Hall Rm 108, Box 19059, Arlington, TX 76019, USA

${ }^{5}$ CTSPS, Clark-Atlanta University, Atlanta, GA 30314, USA
} 
${ }^{6}$ School of Physics and Center for Relativistic Astrophysics, Georgia Institute of Technology, Atlanta, GA 30332, USA

${ }^{7}$ Dept. of Physics, Southern University, Baton Rouge, LA 70813, USA

${ }^{8}$ Dept. of Physics, University of California, Berkeley, CA 94720, USA

${ }^{9}$ Lawrence Berkeley National Laboratory, Berkeley, CA 94720, USA

${ }^{10}$ Institut für Physik, Humboldt-Universität zu Berlin, D-12489 Berlin, Germany

${ }^{11}$ Fakultät für Physik \& Astronomie, Ruhr-Universität Bochum, D-44780 Bochum, Germany

${ }^{12}$ Université Libre de Bruxelles, Science Faculty CP230, B-1050 Brussels, Belgium

${ }^{13}$ Vrije Universiteit Brussel (VUB), Dienst ELEM, B-1050 Brussels, Belgium

${ }^{14}$ Department of Physics and Laboratory for Particle Physics and Cosmology, Harvard University, Cambridge, MA 02138, USA

${ }^{15}$ Dept. of Physics, Massachusetts Institute of Technology, Cambridge, MA 02139, USA

${ }^{16}$ Dept. of Physics and Institute for Global Prominent Research, Chiba University, Chiba 263-8522, Japan

${ }^{17}$ Department of Physics, Loyola University Chicago, Chicago, IL 60660, USA

${ }^{18}$ Dept. of Physics and Astronomy, University of Canterbury, Private Bag 4800, Christchurch, New Zealand

19 Dept. of Physics, University of Maryland, College Park, MD 20742, USA

${ }^{20}$ Dept. of Astronomy, Ohio State University, Columbus, OH 43210, USA

${ }^{21}$ Dept. of Physics and Center for Cosmology and Astro-Particle Physics, Ohio State University, Columbus, OH 43210, USA

${ }^{22}$ Niels Bohr Institute, University of Copenhagen, DK-2100 Copenhagen, Denmark

${ }^{23}$ Dept. of Physics, TU Dortmund University, D-44221 Dortmund, Germany

${ }^{24}$ Dept. of Physics and Astronomy, Michigan State University, East Lansing, MI 48824, USA

${ }^{25}$ Dept. of Physics, University of Alberta, Edmonton, Alberta, Canada T6G 2E1

${ }^{26}$ Erlangen Centre for Astroparticle Physics, Friedrich-Alexander-Universität Erlangen-Nürnberg, D-91058 Erlangen, Germany

${ }^{27}$ Physik-department, Technische Universität München, D-85748 Garching, Germany

${ }^{28}$ Département de physique nucléaire et corpusculaire, Université de Genève, CH-1211 Genève, Switzerland

${ }^{29}$ Dept. of Physics and Astronomy, University of Gent, B-9000 Gent, Belgium

${ }^{30}$ Dept. of Physics and Astronomy, University of California, Irvine, CA 92697, USA

${ }^{31}$ Karlsruhe Institute of Technology, Institute for Astroparticle Physics, D-76021 Karlsruhe, Germany

32 Karlsruhe Institute of Technology, Institute of Experimental Particle Physics, D-76021 Karlsruhe, Germany

${ }^{33}$ Dept. of Physics, Engineering Physics, and Astronomy, Queen's University, Kingston, ON K7L 3N6, Canada

${ }^{34}$ Dept. of Physics and Astronomy, University of Kansas, Lawrence, KS 66045, USA

${ }^{35}$ Department of Physics and Astronomy, UCLA, Los Angeles, CA 90095, USA

${ }^{36}$ Department of Physics, Mercer University, Macon, GA 31207-0001, USA

${ }^{37}$ Dept. of Astronomy, University of Wisconsin-Madison, Madison, WI 53706, USA

${ }^{38}$ Dept. of Physics and Wisconsin IceCube Particle Astrophysics Center, University of Wisconsin-Madison, Madison, WI 53706, USA

${ }^{39}$ Institute of Physics, University of Mainz, Staudinger Weg 7, D-55099 Mainz, Germany

${ }^{40}$ Department of Physics, Marquette University, Milwaukee, WI, 53201, USA

${ }^{41}$ Institut für Kernphysik, Westfälische Wilhelms-Universität Münster, D-48149 Münster, Germany

42 Bartol Research Institute and Dept. of Physics and Astronomy, University of Delaware, Newark, DE 19716, USA

${ }^{43}$ Dept. of Physics, Yale University, New Haven, CT 06520, USA

44 Dept. of Physics, University of Oxford, Parks Road, Oxford OX1 3PU, UK

${ }^{45}$ Dept. of Physics, Drexel University, 3141 Chestnut Street, Philadelphia, PA 19104, USA

${ }^{46}$ Physics Department, South Dakota School of Mines and Technology, Rapid City, SD 57701, USA

47 Dept. of Physics, University of Wisconsin, River Falls, WI 54022, USA

48 Dept. of Physics and Astronomy, University of Rochester, Rochester, NY 14627, USA

${ }^{49}$ Department of Physics and Astronomy, University of Utah, Salt Lake City, UT 84112, USA

${ }^{50}$ Oskar Klein Centre and Dept. of Physics, Stockholm University, SE-10691 Stockholm, Sweden

${ }^{51}$ Dept. of Physics and Astronomy, Stony Brook University, Stony Brook, NY 11794-3800, USA

52 Dept. of Physics, Sungkyunkwan University, Suwon 16419, Korea

${ }^{53}$ Institute of Basic Science, Sungkyunkwan University, Suwon 16419, Korea

${ }^{54}$ Dept. of Physics and Astronomy, University of Alabama, Tuscaloosa, AL 35487, USA

55 Dept. of Astronomy and Astrophysics, Pennsylvania State University, University Park, PA 16802, USA

${ }^{56}$ Dept. of Physics, Pennsylvania State University, University Park, PA 16802, USA

${ }^{57}$ Dept. of Physics and Astronomy, Uppsala University, Box 516, S-75120 Uppsala, Sweden

58 Dept. of Physics, University of Wuppertal, D-42119 Wuppertal, Germany

${ }^{59}$ DESY, D-15738 Zeuthen, Germany

${ }^{60}$ Università di Padova, I-35131 Padova, Italy

${ }^{61}$ National Research Nuclear University, Moscow Engineering Physics Institute (MEPhI), Moscow 115409, Russia

${ }^{62}$ Earthquake Research Institute, University of Tokyo, Bunkyo, Tokyo 113-0032, Japan

\section{Acknowledgements:}

USA - U.S. National Science Foundation-Office of Polar Programs, U.S. National Science Foundation-Physics Division, U.S. Na- 
tional Science Foundation-EPSCoR, Wisconsin Alumni Research Foundation, Center for High Throughput Computing (CHTC) at the University of Wisconsin-Madison, Open Science Grid (OSG), Extreme Science and Engineering Discovery Environment (XSEDE), Frontera computing project at the Texas Advanced Computing Center, U.S. Department of Energy-National Energy Research Scientific Computing Center, Particle astrophysics research computing center at the University of Maryland, Institute for Cyber-Enabled Research at Michigan State University, and Astroparticle physics computational facility at Marquette University; Belgium - Funds for Scientific Research (FRS-FNRS and FWO), FWO Odysseus and Big Science programmes, and Belgian Federal Science Policy Office (Belspo); Germany - Bundesministerium für Bildung und Forschung (BMBF), Deutsche Forschungsgemeinschaft (DFG), Helmholtz Alliance for Astroparticle Physics (HAP), Initiative and Networking Fund of the Helmholtz Association, Deutsches Elektronen Synchrotron (DESY), and High Performance Computing cluster of the RWTH Aachen; Sweden - Swedish Research Council, Swedish Polar Research Secretariat, Swedish National Infrastructure for Computing (SNIC), and Knut and Alice Wallenberg Foundation; Australia - Australian Research Council; Canada - Natural Sciences and Engineering Research Council of Canada, Calcul Québec, Compute Ontario, Canada Foundation for Innovation, WestGrid, and Compute Canada; Denmark - Villum Fonden and Carlsberg Foundation; New Zealand Marsden Fund; Japan - Japan Society for Promotion of Science (JSPS) and Institute for Global Prominent Research (IGPR) of Chiba University; Korea - National Research Foundation of Korea (NRF); Switzerland - Swiss National Science Foundation (SNSF); United Kingdom - Department of Physics, University of Oxford.

\section{KASCADE-Grande Collaboration:}

W. D. Ape ${ }^{1}$, J. C. Arteaga-Velázquez ${ }^{2}$, K. Bekk ${ }^{1}$, M. Bertaina ${ }^{3}$, J. Blümer ${ }^{1,4}$, H. Bozdog ${ }^{1}$, E. Cantoni ${ }^{3,5}$, A. Chiavassa ${ }^{3}$, F. Cossavella ${ }^{4}$, K. Daumiller ${ }^{1}$, V. de Souza ${ }^{7}$, F. Di Pierro ${ }^{3}$, P. Doll ${ }^{1}$, R. Engel ${ }^{1,4}$, D. Fuhrmann ${ }^{8}$, A. Gherghel-Lascu ${ }^{5}$, H. J. Gils ${ }^{1}$, R. Glasstetter ${ }^{8}$, C. Grupen ${ }^{9}$, A. Haungs ${ }^{1}$, D. Heck ${ }^{1}$, J. R. Hörandel ${ }^{10}$, T. Huege ${ }^{1}$, K.-H. Kampert ${ }^{8}$, D. Kang ${ }^{1}$, H. O. Klages ${ }^{1}$, K. Link ${ }^{1}$, P. Luczak $^{11}$, H. J. Mathes ${ }^{1}$, H. J. Mayer ${ }^{1}$, J. Milke ${ }^{1}$, C. Morello ${ }^{6}$, J. Oehlschläger ${ }^{1}$, S. Ostapchenko ${ }^{12}$, T. Pierog ${ }^{1}$, H. Rebel ${ }^{1}$, D. Rivera-Rangel ${ }^{2}$, M. Roth ${ }^{1}$, H. Schieler ${ }^{1}$, S. Schoo ${ }^{1}$, F. G. Schröder ${ }^{1}$, O. Sima ${ }^{13}$, G. Toma ${ }^{5}$, G. C. Trinchero ${ }^{6}$, H. Ulrich ${ }^{1}$, A. Weindl ${ }^{1}$, J. Wochele ${ }^{1}$, J. Zabierowski $^{11}$

${ }^{1}$ Karlsruhe Institute of Technology, Institute for Astroparticle Physics, Karlsruhe, Germany

${ }^{2}$ Universidad Michoacana, Inst. Física y Matemáticas, Morelia, Mexico

${ }^{3}$ Dipartimento di Fisica, Università degli Studi di Torino, Italy

${ }^{4}$ Institut für Experimentelle Teilchenphysik KIT - Karlsruhe Institute of Technology, Germany

${ }^{5}$ Horia Hulubei National Institute of Physics and Nuclear Engineering, Bucharest, Romania

${ }^{6}$ Osservatorio Astrofisico di Torino, INAF Torino, Italy

${ }^{7}$ Universidade São Paulo, Instituto de Física de São Carlos, Brasil

${ }^{8}$ Fachbereich Physik, Universität Wuppertal, Germany

${ }^{9}$ Department of Physics, Siegen University, Germany

${ }^{10}$ Dept. of Astrophysics, Radboud University Nijmegen, The Netherlands

${ }^{11}$ National Centre for Nuclear Research, Department of Astrophysics, Lodz, Poland

12 Frankfurt Institute for Advanced Studies (FIAS), Frankfurt am Main, Germany

${ }^{13}$ Department of Physics, University of Bucharest, Bucharest, Romania

\section{NEVDOD-DECOR Collaboration:}

N. S. Barbashina ${ }^{1}$, A. G. Bogdanov ${ }^{1}$, S. S. Khokhlov ${ }^{1}$, V. V. Kindin ${ }^{1}$, R. P. Kokoulin ${ }^{1}$, K. G. Kompaniets ${ }^{1}$, G. Mannocchi ${ }^{2}$, A. A. Petrukhin $^{1}$, V. V. Shutenko ${ }^{1}$, G. Trinchero ${ }^{2}$, I. I. Yashin ${ }^{1}$, E. A. Yurina ${ }^{1}$, E. A. Zadeba ${ }^{1}$

${ }^{1}$ National Research Nuclear University MEPhI (Moscow Engineering Physics Institute), Moscow, Russia

${ }^{2}$ Osservatorio Astrofisico di Torino - INAF, Torino, Italy

\section{Acknowledgements:}

The work of the NEVOD-DECOR group is supported by the Ministry of Science and Higher Education of the Russian Federation, project "Fundamental problems of cosmic rays and dark matter", No. 0723-2020-0040.

\section{Pierre Auger Collaboration:}

P. Abreu ${ }^{72}$, M. Aglietta ${ }^{5,52}$, J.M. Albury ${ }^{13}$, I. Allekotte ${ }^{1}$, A. Almela ${ }^{8,12}$, J. Alvarez-Muñiz ${ }^{79}$, R. Alves Batista ${ }^{80}$, G.A. Anastasi ${ }^{63,52}$, L. Anchordoqui ${ }^{87}$, B. Andrada ${ }^{8}$, S. Andringa ${ }^{72}$, C. Aramo ${ }^{50}$, P.R. Araújo Ferreira ${ }^{42}$, J. C. Arteaga Velázquez ${ }^{67}$, H. Asorey ${ }^{8}$, P. Assis ${ }^{72}$, G. Avila ${ }^{11}$, A.M. Badescu ${ }^{75}$, A. Bakalova ${ }^{32}$, A. Balaceanu ${ }^{73}$, F. Barbato ${ }^{45,46}$, R.J. Barreira Luz $^{72}$, K.H. Becker ${ }^{38}$, J.A. Bellido ${ }^{13,69}$, C. Berat ${ }^{36}$, M.E. Bertaina ${ }^{63,52}$, X. Bertou ${ }^{1}$, P.L. Biermann ${ }^{b}$, V. Binet ${ }^{6}$, K. Bismark ${ }^{39,8}$, T. Bister ${ }^{42}$, J. Biteau ${ }^{37}$, J. Blazek ${ }^{32}$, C. Bleve ${ }^{36}$, M. Boháčová ${ }^{32}$, D. Boncioli ${ }^{57,46}$, C. Bonifazi ${ }^{9,26}$, L. Bonneau Arbeletche ${ }^{21}$, N. Borodai ${ }^{70}$, A.M. Botti ${ }^{8}$, J. Brack ${ }^{d}$, T. Bretz ${ }^{42}$, P.G. Brichetto Orchera ${ }^{8}$, F.L. Briechle ${ }^{42}$, P. Buchholz ${ }^{44}$, A. Bueno ${ }^{78}$, S. Buitink ${ }^{15}$, M. Buscemi ${ }^{47}$, M. Büsken ${ }^{39,8}$, K.S. CaballeroMora $^{66}$, L. Caccianiga ${ }^{59,49}$, F. Canfora ${ }^{80,81}$, I. Caracas $^{38}$, J.M. Carceller ${ }^{78}$, R. Caruso ${ }^{58,47}$, A. Castellina ${ }^{54,52}$, F. Catalani ${ }^{19}$, 


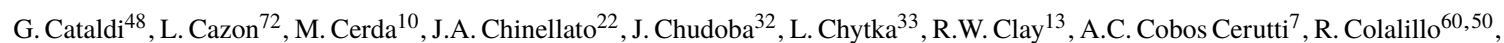
A. Coleman ${ }^{93}$, M.R. Coluccia ${ }^{48}$, R. Conceição ${ }^{72}$, A. Condorelli ${ }^{45,46}$, G. Consolati ${ }^{49,55}$, F. Contreras ${ }^{11}$, F. Convenga ${ }^{56,48}$, D. Correia dos Santos $^{28}$, C.E. Covault $^{85}$, S. Dasso ${ }^{5,3}$, K. Daumiller ${ }^{41}$, B.R. Dawson ${ }^{13}$, J.A. Day ${ }^{13}$, R.M. de Almeida ${ }^{28}$, J. de Jesús ${ }^{8,41}$, S.J. de Jong ${ }^{80,81}$, G. De Mauro ${ }^{80,81}$, J.R.T. de Mello Neto $^{26,27}$, I. De Mitri ${ }^{45,46}$, J. de Oliveira ${ }^{18}$, D. de Oliveira Franco ${ }^{22}$, F. de Palma $^{56,48}$, V. de Souza ${ }^{20}$, E. De Vito ${ }^{56,48}$, M. del Río ${ }^{11}$, O. Deligny ${ }^{34}$, L. Deval ${ }^{41,8}$, A. di Matteo ${ }^{52}$, C. Dobrigkeit ${ }^{22}$, J.C. D’Olivo ${ }^{68}$, L.M. Domingues Mendes ${ }^{72}$, R.C. dos Anjos ${ }^{25}$, D. dos $\operatorname{Santos}^{28}$, M.T. Dova ${ }^{4}$, J. Ebr $^{32}$, R. Engel ${ }^{39,41}$, I. Epicoco ${ }^{56,48}$, M. Erdmann ${ }^{42}$, C.O. Escobar ${ }^{a}$, A. Etchegoyen ${ }^{8,12}$, H. Falcke $80,82,81$, J. Farmer ${ }^{92}$, G. Farrar ${ }^{90}$, A.C. Fauth ${ }^{22}$, N. Fazzini ${ }^{a}$, F. Feldbusch ${ }^{40}$, F. Fenu $^{54,52}$, B. Fick ${ }^{89}$, J.M. Figueira ${ }^{8}$, A. Filipčič 77,76 , T. Fitoussi ${ }^{41}$, T. Fodran ${ }^{80}$, M.M. Freire ${ }^{6}$, T. Fujii ${ }^{22, e}$, A. Fuster ${ }^{8,12}$, C. Galea ${ }^{80}$, C. Galelli ${ }^{59,49}$, B. García ${ }^{7}$, A.L. Garcia Vegas ${ }^{42}$, H. Gemmeke ${ }^{40}$, F. Gesualdi ${ }^{8,41}$, A. Gherghel-Lascu ${ }^{73}$, P.L. Ghia ${ }^{34}$, U. Giaccari ${ }^{80}$, M. Giammarchi ${ }^{49}$, J. Glombitza ${ }^{42}$, F. Gobbi ${ }^{10}$, F. Gollan ${ }^{8}$, G. Golup ${ }^{1}$, M. Gómez Berisso ${ }^{1}$, P.F. Gómez Vitale ${ }^{11}$, J.P. Gongora ${ }^{11}$, J.M. González ${ }^{1}$, N. González ${ }^{14}$, I. Goos ${ }^{1,41}$, D. Góra ${ }^{70}$, A. Gorgi ${ }^{54,52}$, M. Gottowik ${ }^{38}$, T.D. Grubb ${ }^{13}$, F. Guarino ${ }^{60,50}$, G.P. Guedes ${ }^{23}$, E. Guido ${ }^{52,63}$, S. Hahn ${ }^{41,8}$, P. Hamal ${ }^{32}$, M.R. Hampel ${ }^{8}$, P. Hansen ${ }^{4}$, D. Harari ${ }^{1}$, V.M. Harvey ${ }^{13}$, A. Haungs ${ }^{41}$, T. Hebbeker ${ }^{42}$, D. Heck ${ }^{41}$, G.C. Hill ${ }^{13}$, C. Hojvat ${ }^{a}$, J.R. Hörandel ${ }^{80,81}$, P. Horvath ${ }^{33}$, M. Hrabovský33, T. Huege ${ }^{41,15}$, A. Insolia ${ }^{58,47}$, P.G. Isar ${ }^{74}$, P. Janecek ${ }^{32}$, J.A. Johnsen ${ }^{86}$, J. Jurysek ${ }^{32}$, A. Kääpä ${ }^{38}$, K.H. Kampert ${ }^{38}$, N. Karastathis ${ }^{41}$, B. Keilhauer ${ }^{41}$, J. Kemp ${ }^{42}$, A. Khakurdikar ${ }^{80}$, V.V. Kizakke Covilakam ${ }^{8,41}$, H.O. Klages ${ }^{41}$, M. Kleifges ${ }^{40}$, J. Kleinfeller ${ }^{10}$, M. Köpke ${ }^{39}$, N. Kunka ${ }^{40}$, B.L. Lago ${ }^{17}$, R.G. Lang ${ }^{20}$, N. Langner ${ }^{42}$, M.A. Leigui de Oliveira ${ }^{24}$, V. Lenok ${ }^{41}$, A. Letessier-Selvon ${ }^{35}$, I. Lhenry-Yvon ${ }^{34}$, D. Lo Presti ${ }^{58,47}$, L. Lopes ${ }^{72}$, R. López ${ }^{64}$, L. Lu ${ }^{94}$, Q. Luce ${ }^{39}$, J.P. Lundquist ${ }^{76}$, A. Machado Payeras ${ }^{22}$, G. Mancarella ${ }^{56,48}$, D. Mandat ${ }^{32}$, B.C. Manning ${ }^{13}$, J. Manshanden ${ }^{43}$, P. Mantsch ${ }^{a}$, S. Marafico ${ }^{34}$, A.G. Mariazzi ${ }^{4}$, I.C. Mariş ${ }^{14}$, G. Marsella ${ }^{61,47}$, D. Martello ${ }^{56,48}$, S. Martinelli ${ }^{41,8}$, O. Martínez Bravo ${ }^{64}$, M. Mastrodicasa ${ }^{57,46}$, H.J. Mathes ${ }^{41}$, J. Matthews ${ }^{88}$, G. Matthiae ${ }^{62,51}$, E. Mayotte ${ }^{38}$, P.O. Mazur ${ }^{a}$, G. MedinaTanco $^{68}$, D. Melo ${ }^{8}$, A. Menshikov ${ }^{40}$, K.-D. Merenda ${ }^{86}$, S. Michal ${ }^{33}$, M.I. Micheletti ${ }^{6}$, L. Miramonti ${ }^{59,49}$, S. Mollerach ${ }^{1}$, F. Montanet ${ }^{36}$, C. Morello ${ }^{54,52}$, M. Mostafá ${ }^{91}$, A.L. Müller ${ }^{8}$, M.A. Muller ${ }^{22}$, K. Mulrey ${ }^{15}$, R. Mussa ${ }^{52}$, M. Muzio ${ }^{90}$, W.M. Namasaka ${ }^{38}$, A. NasrEsfahani $^{38}$, L. Nellen ${ }^{68}$, M. Niculescu-Oglinzanu ${ }^{73}$, M. Niechciol ${ }^{44}$, D. Nitz ${ }^{89}$, D. Nosek ${ }^{31}$, V. Novotny ${ }^{31}$, L. Nožka ${ }^{33}$, A Nucita ${ }^{56,48}$, L.A. Núñez ${ }^{30}$, M. Palatka ${ }^{32}$, J. Pallotta ${ }^{2}$, P. Papenbreer ${ }^{38}$, G. Parente ${ }^{79}$, A. Parra ${ }^{64}$, J. Pawlowsky ${ }^{38}$, M. Pech ${ }^{32}$, F. Pedreira ${ }^{79}$, J. Pȩkala ${ }^{70}$, R. Pelayo ${ }^{65}$, J. Peña-Rodriguez ${ }^{30}$, E.E. Pereira Martins ${ }^{39,8}$, J. Perez Armand ${ }^{21}$, C. Pérez Bertolli ${ }^{8,41}$, M. Perlin ${ }^{8,41}$, L. Perrone ${ }^{56,48}$, S. Petrera ${ }^{45,46}$, T. Pierog ${ }^{41}$, M. Pimenta ${ }^{72}$, V. Pirronello ${ }^{58,47}$, M. Platino ${ }^{8}$, B. Pont ${ }^{80}$, M. Pothast ${ }^{81,80}$, P. Privitera ${ }^{92}$, M. Prouza ${ }^{32}$, A. Puyleart ${ }^{89}$, S. Querchfeld ${ }^{38}$, J. Rautenberg ${ }^{38}$, D. Ravignani ${ }^{8}$, M. Reininghaus ${ }^{41,8}$, J. Ridky ${ }^{32}$, F. Riehn ${ }^{72}$, M. Risse ${ }^{44}$, V. Rizi ${ }^{57,46}$, W. Rodrigues de Carvalho ${ }^{21}$, J. Rodriguez Rojo ${ }^{11}$, M.J. Roncoroni ${ }^{8}$, S. Rossoni ${ }^{43}$, M. Roth ${ }^{41}$, E. Roulet ${ }^{1}$, A.C. Rovero ${ }^{5}$, P. Rueh ${ }^{44}$, A. Saftoiu ${ }^{73}$, F. Salamida ${ }^{57,46}$, H. Salazar ${ }^{64}$, G. Salina ${ }^{51}$, J.D. Sanabria Gomez ${ }^{30}$, F. Sánchez ${ }^{8}$, E.M. Santos ${ }^{21}$, E. Santos ${ }^{32}$, F. Sarazin ${ }^{86}$, R. Sarmento ${ }^{72}$, C. Sarmiento-Cano ${ }^{8}$, R. Sato ${ }^{11}$, P. Savina ${ }^{56,48,34,94}$, C.M. Schäfer ${ }^{41}$, V. Scherini ${ }^{56,48}$, H. Schieler ${ }^{41}$, M. Schimassek ${ }^{39,8}$, M. Schimp ${ }^{38}$, F. Schlüter ${ }^{41,8}$, D. Schmidt ${ }^{39}$, O. Scholten ${ }^{84,15}$, P. Schovánek ${ }^{32}$, F.G. Schröder ${ }^{93,41}$, S. Schröder ${ }^{38}$, J. Schulte ${ }^{42}$, S.J. Sciutto ${ }^{4}$, M. Scornavacche ${ }^{8,41}$, A. Segreto ${ }^{53,47}$, S. Sehgal ${ }^{38}$, R.C. Shellard ${ }^{16}$, G. Sigl ${ }^{43}$, G. Silli ${ }^{8,41}$, O. Sima ${ }^{73, f}$, R. Šmída ${ }^{92}$, P. Sommers ${ }^{91}$, J.F. Soriano ${ }^{87}$, J. Souchard ${ }^{36}$, R. Squartini ${ }^{10}$, M. Stadelmaier ${ }^{41,8}$, D. Stanca ${ }^{73}$, S. Stanič ${ }^{76}$, J. Stasielak ${ }^{70}$ P. Stassi ${ }^{36}$, A. Streich ${ }^{39,8}$, M. Suárez-Durán ${ }^{14}$, T. Sudholz ${ }^{13}$, T. Suomijärvi ${ }^{37}$, A.D. Supanitsky ${ }^{8}$, Z. Szadkowski ${ }^{71}$, A. Tapia ${ }^{29}$, C. Taricco $^{63,52}$, C. Timmermans ${ }^{81,80}$, O. Tkachenko ${ }^{41}$, P. Tobiska ${ }^{32}$, C.J. Todero Peixoto ${ }^{19}$, B. Tomé ${ }^{72}$, Z. Torrès ${ }^{36}$, A. Travaini ${ }^{10}$, P. Travnicek ${ }^{32}$, C. Trimarelli ${ }^{57,46}$, M. Tueros ${ }^{4}$, R. Ulrich ${ }^{41}$, M. Unger ${ }^{41}$, L. Vaclavek ${ }^{33}$, M. Vacula ${ }^{33}$, J.F. Valdés Galicia ${ }^{68}$, L. Valore ${ }^{60,50}$, E. Varela ${ }^{64}$, A. Vásquez-Ramírez ${ }^{30}$, D. Veberic ${ }^{41}$, C. Ventura ${ }^{27}$, I.D. Vergara Quispe ${ }^{4}$, V. Verzi ${ }^{51}$, J. Vicha $^{32}$, J. Vink $^{83}$, S. Vorobiov $^{76}$, H. Wahlberg ${ }^{4}$, C. Watanabe ${ }^{26}$, A.A. Watson ${ }^{c}$, M. Weber ${ }^{40}$, A. Weindl ${ }^{41}$, L. Wiencke ${ }^{86}$, H. Wilczyński $^{70}$, M. Wirtz $^{42}$, D. Wittkowski $^{38}$, B. Wundheiler ${ }^{8}$, A. Yushkov ${ }^{32}$, O. Zapparrata ${ }^{14}$, E. Zas ${ }^{79}$, D. Zavrtanik ${ }^{76,77}$, M. Zavrtanik ${ }^{77,76}$, L. Zehrer ${ }^{76}$

${ }^{1}$ Centro Atómico Bariloche and Instituto Balseiro (CNEA-UNCuyo-CONICET), San Carlos de Bariloche, Argentina

2 Centro de Investigaciones en Láseres y Aplicaciones, CITEDEF and CONICET, Villa Martelli, Argentina

${ }^{3}$ Departamento de Física and Departamento de Ciencias de la Atmósfera y los Océanos, FCEyN, Universidad de Buenos Aires and CONICET, Buenos Aires, Argentina

${ }^{4}$ IFLP, Universidad Nacional de La Plata and CONICET, La Plata, Argentina

${ }^{5}$ Instituto de Astronomía y Física del Espacio (IAFE, CONICET-UBA), Buenos Aires, Argentina

${ }^{6}$ Instituto de Física de Rosario (IFIR) - CONICET/U.N.R. and Facultad de Ciencias Bioquímicas y Farmacéuticas U.N.R., Rosario, Argentina

${ }^{7}$ Instituto de Tecnologías en Detección y Astropartículas (CNEA, CONICET, UNSAM), and Universidad Tecnológica Nacional Facultad Regional Mendoza (CONICET/CNEA), Mendoza, Argentina

${ }^{8}$ Instituto de Tecnologías en Detección y Astropartículas (CNEA, CONICET, UNSAM), Buenos Aires, Argentina

${ }^{9}$ International Center of Advanced Studies and Instituto de Ciencias Físicas, ECyT-UNSAM and CONICET, Campus Miguelete - San Martín, Buenos Aires, Argentina

10 Observatorio Pierre Auger, Malargüe, Argentina

11 Observatorio Pierre Auger and Comisión Nacional de Energía Atómica, Malargüe, Argentina

12 Universidad Tecnológica Nacional - Facultad Regional Buenos Aires, Buenos Aires, Argentina

${ }^{13}$ University of Adelaide, Adelaide, S.A., Australia

14 Université Libre de Bruxelles (ULB), Brussels, Belgium

15 Vrije Universiteit Brussels, Brussels, Belgium

16 Centro Brasileiro de Pesquisas Fisicas, Rio de Janeiro, RJ, Brazil

${ }^{17}$ Centro Federal de Educação Tecnológica Celso Suckow da Fonseca, Nova Friburgo, Brazil

18 Instituto Federal de Educação, Ciência e Tecnologia do Rio de Janeiro (IFRJ), Brazil 
${ }^{19}$ Universidade de São Paulo, Escola de Engenharia de Lorena, Lorena, SP, Brazil

${ }^{20}$ Universidade de São Paulo, Instituto de Física de São Carlos, São Carlos, SP, Brazil

${ }^{21}$ Universidade de São Paulo, Instituto de Física, São Paulo, SP, Brazil

${ }^{22}$ Universidade Estadual de Campinas, IFGW, Campinas, SP, Brazil

${ }^{23}$ Universidade Estadual de Feira de Santana, Feira de Santana, Brazil

${ }^{24}$ Universidade Federal do ABC, Santo André, SP, Brazil

${ }^{25}$ Universidade Federal do Paraná, Setor Palotina, Palotina, Brazil

${ }^{26}$ Universidade Federal do Rio de Janeiro, Instituto de Física, Rio de Janeiro, RJ, Brazil

${ }^{27}$ Universidade Federal do Rio de Janeiro (UFRJ), Observatório do Valongo, Rio de Janeiro, RJ, Brazil

${ }^{28}$ Universidade Federal Fluminense, EEIMVR, Volta Redonda, RJ, Brazil

${ }^{29}$ Universidad de Medellín, Medellín, Colombia

${ }^{30}$ Universidad Industrial de Santander, Bucaramanga, Colombia

${ }^{31}$ Charles University, Faculty of Mathematics and Physics, Institute of Particle and Nuclear Physics, Prague, Czech Republic

32 Institute of Physics of the Czech Academy of Sciences, Prague, Czech Republic

${ }^{33}$ Palacky University, RCPTM, Olomouc, Czech Republic

${ }^{34}$ CNRS/IN2P3, IJCLab, Université Paris-Saclay, Orsay, France

${ }^{35}$ Laboratoire de Physique Nucléaire et de Hautes Energies (LPNHE), Sorbonne Université, Université de Paris, CNRS-IN2P3, Paris, France

${ }^{36}$ Univ. Grenoble Alpes, CNRS, Grenoble Institute of Engineering Univ. Grenoble Alpes, LPSC-IN2P3, 38000 Grenoble, France

${ }^{37}$ Université Paris-Saclay, CNRS/IN2P3, IJCLab, Orsay, France

${ }^{38}$ Bergische Universität Wuppertal, Department of Physics, Wuppertal, Germany

${ }^{39}$ Karlsruhe Institute of Technology (KIT), Institute for Experimental Particle Physics, Karlsruhe, Germany

${ }^{40}$ Karlsruhe Institute of Technology (KIT), Institut für Prozessdatenverarbeitung und Elektronik, Karlsruhe, Germany

${ }^{41}$ Karlsruhe Institute of Technology (KIT), Institute for Astroparticle Physics, Karlsruhe, Germany

${ }^{42}$ RWTH Aachen University, III. Physikalisches Institut A, Aachen, Germany

${ }^{43}$ Universität Hamburg, II. Institut für Theoretische Physik, Hamburg, Germany

${ }^{44}$ Universität Siegen, Department Physik - Experimentelle Teilchenphysik, Siegen, Germany

${ }^{45}$ Gran Sasso Science Institute, L'Aquila, Italy

${ }^{46}$ INFN Laboratori Nazionali del Gran Sasso, Assergi (L’Aquila), Italy

${ }^{47}$ INFN, Sezione di Catania, Catania, Italy

${ }^{48}$ INFN, Sezione di Lecce, Lecce, Italy

${ }^{49}$ INFN, Sezione di Milano, Milano, Italy

${ }^{50}$ INFN, Sezione di Napoli, Napoli, Italy

${ }^{51}$ INFN, Sezione di Roma "Tor Vergata”, Roma, Italy

${ }^{52}$ INFN, Sezione di Torino, Torino, Italy

${ }^{53}$ Istituto di Astrofisica Spaziale e Fisica Cosmica di Palermo (INAF), Palermo, Italy

${ }^{54}$ Osservatorio Astrofisico di Torino (INAF), Torino, Italy

${ }^{55}$ Politecnico di Milano, Dipartimento di Scienze e Tecnologie Aerospaziali, Milano, Italy

${ }^{56}$ Università del Salento, Dipartimento di Matematica e Fisica “E. De Giorgi”, Lecce, Italy

${ }^{57}$ Università dell'Aquila, Dipartimento di Scienze Fisiche e Chimiche, L'Aquila, Italy

${ }^{58}$ Università di Catania, Dipartimento di Fisica e Astronomia, Catania, Italy

${ }^{59}$ Università di Milano, Dipartimento di Fisica, Milano, Italy

${ }^{60}$ Università di Napoli "Federico II", Dipartimento di Fisica "Ettore Pancini", Napoli, Italy

${ }^{61}$ Università di Palermo, Dipartimento di Fisica e Chimica "E. Segrè", Palermo, Italy

${ }^{62}$ Università di Roma "Tor Vergata", Dipartimento di Fisica, Roma, Italy

${ }^{63}$ Università Torino, Dipartimento di Fisica, Torino, Italy

${ }^{64}$ Benemérita Universidad Autónoma de Puebla, Puebla, México

${ }^{65}$ Unidad Profesional Interdisciplinaria en Ingeniería y Tecnologías Avanzadas del Instituto Politécnico Nacional (UPIITA-IPN), México, D.F., México

${ }^{66}$ Universidad Autónoma de Chiapas, Tuxtla Gutiérrez, Chiapas, México

${ }^{67}$ Universidad Michoacana de San Nicolás de Hidalgo, Morelia, Michoacán, México

${ }^{68}$ Universidad Nacional Autónoma de México, México, D.F., México

${ }^{69}$ Universidad Nacional de San Agustin de Arequipa, Facultad de Ciencias Naturales y Formales, Arequipa, Peru

${ }^{70}$ Institute of Nuclear Physics PAN, Krakow, Poland

${ }^{71}$ University of Łódź, Faculty of High-Energy Astrophysics,Lódź, Poland

72 Laboratório de Instrumentação e Física Experimental de Partículas - LIP and Instituto Superior Técnico - IST, Universidade de Lisboa - UL, Lisboa, Portugal

73 "Horia Hulubei" National Institute for Physics and Nuclear Engineering, Bucharest-Magurele, Romania

${ }^{74}$ Institute of Space Science, Bucharest-Magurele, Romania

${ }^{75}$ University Politehnica of Bucharest, Bucharest, Romania

${ }^{76}$ Center for Astrophysics and Cosmology (CAC), University of Nova Gorica, Nova Gorica, Slovenia 
77 Experimental Particle Physics Department, J. Stefan Institute, Ljubljana, Slovenia

${ }^{78}$ Universidad de Granada and C.A.F.P.E., Granada, Spain

${ }^{79}$ Instituto Galego de Física de Altas Enerxías (IGFAE), Universidade de Santiago de Compostela, Santiago de Compostela, Spain

${ }^{80}$ IMAPP, Radboud University Nijmegen, Nijmegen, The Netherlands

${ }^{81}$ Nationaal Instituut voor Kernfysica en Hoge Energie Fysica (NIKHEF), Science Park, Amsterdam, The Netherlands

82 Stichting Astronomisch Onderzoek in Nederland (ASTRON), Dwingeloo, The Netherlands

${ }^{83}$ Universiteit van Amsterdam, Faculty of Science, Amsterdam, The Netherlands

84 University of Groningen, Kapteyn Astronomical Institute, Groningen, The Netherlands

85 Case Western Reserve University, Cleveland, OH, USA

86 Colorado School of Mines, Golden, CO, USA

${ }^{87}$ Department of Physics and Astronomy, Lehman College, City University of New York, Bronx, NY, USA

${ }^{88}$ Louisiana State University, Baton Rouge, LA, USA

${ }^{89}$ Michigan Technological University, Houghton, MI, USA

${ }^{90}$ New York University, New York, NY, USA

91 Pennsylvania State University, University Park, PA, USA

92 University of Chicago, Enrico Fermi Institute, Chicago, IL, USA

${ }^{93}$ University of Delaware, Department of Physics and Astronomy, Bartol Research Institute, Newark, DE, USA

94 University of Wisconsin-Madison, Department of Physics and WIPAC, Madison, WI, USA

$a$ Fermi National Accelerator Laboratory, Fermilab, Batavia, IL, USA

$b$ Max-Planck-Institut für Radioastronomie, Bonn, Germany

$c$ School of Physics and Astronomy, University of Leeds, Leeds, United Kingdom

${ }^{d}$ Colorado State University, Fort Collins, CO, USA

$e$ now at Hakubi Center for Advanced Research and Graduate School of Science, Kyoto University, Kyoto, Japan

$f$ also at University of Bucharest, Physics Department, Bucharest, Romania

\section{Acknowledgements:}

The successful installation, commissioning, and operation of the Pierre Auger Observatory would not have been possible without the strong commitment and effort from the technical and administrative staff in Malargüe. We are very grateful to the following agencies and organizations for financial support:

Argentina - Comisión Nacional de Energía Atómica; Agencia Nacional de Promoción Científica y Tecnológica (ANPCyT); Consejo Nacional de Investigaciones Científicas y Técnicas (CONICET); Gobierno de la Provincia de Mendoza; Municipalidad de Malargüe; NDM Holdings and Valle Las Leñas; in gratitude for their continuing cooperation over land access; Australia - the Australian Research Council; Belgium - Fonds de la Recherche Scientifique (FNRS); Research Foundation Flanders (FWO); Brazil - Conselho Nacional de Desenvolvimento Científico e Tecnológico (CNPq); Financiadora de Estudos e Projetos (FINEP); Fundação de Amparo à Pesquisa do Estado de Rio de Janeiro (FAPERJ); São Paulo Research Foundation (FAPESP) Grants No. 2019/10151-2, No. 2010/07359-6 and No. 1999/05404-3; Ministério da Ciência, Tecnologia, Inovações e Comunicações (MCTIC); Czech Republic - Grant No. MSMT CR LTT18004, LM2015038, LM2018102, CZ.02.1.01/0.0/0.0/16_013/0001402, CZ.02.1.01/0.0/0.0/18_046/0016010 and CZ.02.1.01/0.0/0 .0/17_049/0008422; France - Centre de Calcul IN2P3/CNRS; Centre National de la Recherche Scientifique (CNRS); Conseil Régional Ile-de-France; Département Physique Nucléaire et Corpusculaire (PNC-IN2P3/CNRS); Département Sciences de 1'Univers (SDUINSU/CNRS); Institut Lagrange de Paris (ILP) Grant No. LABEX ANR-10-LABX-63 within the Investissements d'Avenir Programme Grant No. ANR-11-IDEX-0004-02; Germany - Bundesministerium für Bildung und Forschung (BMBF); Deutsche Forschungsgemeinschaft (DFG); Finanzministerium Baden-Württemberg; Helmholtz Alliance for Astroparticle Physics (HAP); Helmholtz-Gemeinschaft Deutscher Forschungszentren (HGF); Ministerium für Innovation, Wissenschaft und Forschung des Landes Nordrhein-Westfalen; Ministerium für Wissenschaft, Forschung und Kunst des Landes Baden-Württemberg; Italy - Istituto Nazionale di Fisica Nucleare (INFN); Istituto Nazionale di Astrofisica (INAF); Ministero dell' Istruzione, dell'Universitá e della Ricerca (MIUR); CETEMPS Center of Excellence; Ministero degli Affari Esteri (MAE); México - Consejo Nacional de Ciencia y Tecnología (CONACYT) No. 167733; Universidad Nacional Autónoma de México (UNAM); PAPIIT DGAPA-UNAM; The Netherlands - Ministry of Education, Culture and Science; Netherlands Organisation for Scientific Research (NWO); Dutch national e-infrastructure with the support of SURF Cooperative; Poland - Ministry of Education and Science, grant No. DIR/WK/2018/11; National Science Centre, Grants No. 2016/22/M/ST9/00198, 2016/23/B/ST9/01635, and 2020/39/B/ST9/01398; Portugal - Portuguese national funds and FEDER funds within Programa Operacional Factores de Competitividade through Fundação para a Ciência e a Tecnologia (COMPETE); Romania - Ministry of Research, Innovation and Digitization, CNCS/CCCDI - UEFISCDI, projects PN19150201/16N/2019, PN1906010, TE128 and PED289, within PNCDI III; Slovenia - Slovenian Research Agency, grants P1-0031, P1-0385, I0-0033, N1-0111; Spain - Ministerio de Economía, Industria y Competitividad (FPA2017-85114-P and PID2019-104676GB-C32), Xunta de Galicia (ED431C 2017/07), Junta de Andalucía (SOMM17/6104/UGR, P18-FR-4314) Feder Funds, RENATA Red Nacional Temática de Astropartículas (FPA2015-68783-REDT) and María de Maeztu Unit of Excellence (MDM-2016-0692); USA - Department of Energy, Contracts No. DE-AC02-07CH11359, No. DE-FR02-04ER41300, No. DE-FG02-99ER41107 and No. DE-SC0011689; National Science Foundation, Grant No. 0450696; The Grainger Foundation; Marie Curie-IRSES/EPLANET; European Particle Physics Latin American Network; and UNESCO. 


\section{SUGAR Collaboration:}

N. N. Kalmykov ${ }^{1}$, I. S. Karpikov ${ }^{2}$, G. I. Rubtsov ${ }^{2}$, S. V. Troitsky², J. Ulrichs ${ }^{3}$

${ }^{1}$ D.V. Skobeltsyn Institute of Nuclear Physics, M.V. Lomonosov Moscow State University, Moscow 119991, Russia

2 Institute for Nuclear Research of the Russian Academy of Sciences, Moscow, Russia

3 jusyd19@gmail.com

\section{Telescope Array Collaboration:}

R. U. Abbasi ${ }^{1}$, M. Abe ${ }^{2}$, T. Abu-Zayyad ${ }^{1,3}$, M. Allen ${ }^{3}$, Y. Arai ${ }^{4}$, E. Barcikowski ${ }^{3}$, J. W. Belz ${ }^{3}$, D. R. Bergman ${ }^{3}$, S. A. Blake, I. Buckland ${ }^{3}$, R. $\mathrm{Cady}^{3}$, B. G. Cheon ${ }^{5}$, J. Chiba ${ }^{6}$, M. Chikawa ${ }^{7}$, T. Fujii ${ }^{8}$, K. Fujisue ${ }^{7}$, K. Fujita ${ }^{4}$, R. Fujiwara ${ }^{4}$, M. Fukushima ${ }^{7,9}$, R. Fukushima ${ }^{4}$, G. Furlich ${ }^{3}$, R. Gonzalez ${ }^{3}$, W. Hanlon $^{3}$, M. Hayashi ${ }^{10}$, N. Hayashida ${ }^{11}$, K. Hibino ${ }^{11}$, R. Higuchi ${ }^{7}$, K. Honda ${ }^{12}$, D. Ikeda ${ }^{11}$, T. Inadomi ${ }^{13}$, N. Inoue ${ }^{2}$, T. Ishii ${ }^{12}$, H. Ito ${ }^{14}$, D. Ivanov ${ }^{3}$, H. Iwakura ${ }^{13}$, H. M. Jeong ${ }^{15}$, S. Jeong ${ }^{15}$, C. C. H. Jui ${ }^{3}$, K. Kadota ${ }^{16}$, F. Kakimoto ${ }^{11}$, O. Kalashev ${ }^{17}$, K. Kasahara ${ }^{18}$, S. Kasami ${ }^{19}$, H. Kawai ${ }^{20}$, S. Kawakami ${ }^{4}$, S. Kawana ${ }^{2}$, K. Kawata ${ }^{7}$, E. Kido ${ }^{14}$, H. B. Kim ${ }^{5}$, J. H. Kim ${ }^{3}$, J. H. Kim ${ }^{3}$, M. H. Kim ${ }^{15}$, S. W. Kim ${ }^{15}$, Y. Kimura ${ }^{4}$, S. Kishigami ${ }^{4}$, Y. Kubota ${ }^{13}$, S. Kurisu ${ }^{13}$, V. Kuzmin ${ }^{17}$, M. Kuznetsov ${ }^{17,21}$, Y. J. Kwon ${ }^{22}$, K. H. Lee ${ }^{15}$, B. Lubsandorzhiev ${ }^{17}$, J. P. Lundquist ${ }^{3,23}$, K. Machida ${ }^{12}$, H. Matsumiya ${ }^{4}$, T. Matsuyama ${ }^{4}$, J. N. Matthews ${ }^{3}$, R. Mayta ${ }^{4}$, M. Minamino ${ }^{4}$, K. Mukai ${ }^{12}$, I. Myers ${ }^{3}$, S. Nagataki ${ }^{14}$, K. Nakai ${ }^{4}$, R. Nakamura ${ }^{13}$,

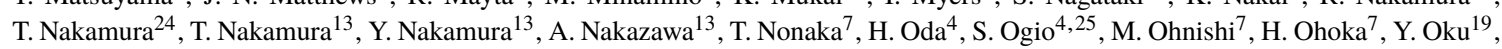
T. Okuda ${ }^{26}$, Y. Omura ${ }^{4}$, M. Ono ${ }^{14}$, R. Onogi ${ }^{4}$, A. Oshima ${ }^{4}$, S. Ozawa ${ }^{27}$, I.H. Park ${ }^{15}$, M. Potts ${ }^{3}$, M.S. Pshirkov ${ }^{17,28}$, J. Remington ${ }^{3}$, D. C. Rodriguez ${ }^{3}$, G. I. Rubtsov ${ }^{17}$, D. Ryu ${ }^{29}$, H. Sagawa $^{7}$, R. Sahara ${ }^{4}$, Y. Saito ${ }^{13}$, N. Sakaki ${ }^{7}$, T. Sako ${ }^{7}$, N. Sakurai ${ }^{4}$, K. Sano ${ }^{13}$, K. Sato ${ }^{4}$, T. Seki ${ }^{13}$, K. Sekino ${ }^{7}$, P.D. Shah ${ }^{3}$, Y. Shibasaki ${ }^{13}$, F. Shibata ${ }^{12}$, N. Shibata ${ }^{19}$, T. Shibata ${ }^{7}$, H. Shimodaira ${ }^{7}$, B. K. Shin ${ }^{29}$, H. S. Shin ${ }^{7}$, D. Shinto ${ }^{19}$, J. D. Smith ${ }^{3}$, P. Sokolsky ${ }^{3}$, N. Sone ${ }^{13}$, B. T. Stokes ${ }^{3}$, T. A. Stroman ${ }^{3}$, T. Suzawa ${ }^{2}$, Y. Takagi ${ }^{4}$, Y. Takahashi ${ }^{4}$,

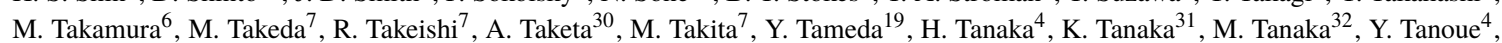

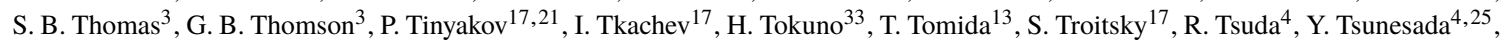

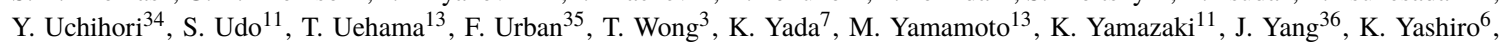
F. Yoshida ${ }^{19}$, Y. Yoshioka $^{13}$, Y. Zhezher ${ }^{7,17}$, and Z. Zundel ${ }^{3}$

${ }^{1}$ Department of Physics, Loyola University Chicago, Chicago, Illinois, USA

2 The Graduate School of Science and Engineering, Saitama University, Saitama, Saitama, Japan

${ }^{3}$ High Energy Astrophysics Institute and Department of Physics and Astronomy, University of Utah, Salt Lake City, Utah, USA

${ }^{4}$ Graduate School of Science, Osaka City University, Osaka, Osaka, Japan

${ }^{5}$ Department of Physics and The Research Institute of Natural Science, Hanyang University, Seongdong-gu, Seoul, Korea

${ }^{6}$ Department of Physics, Tokyo University of Science, Noda, Chiba, Japan

${ }^{7}$ Institute for Cosmic Ray Research, University of Tokyo, Kashiwa, Chiba, Japan

${ }^{8}$ The Hakubi Center for Advanced Research and Graduate School of Science, Kyoto University, Kitashirakawa-Oiwakecho, Sakyo-ku, Kyoto, Japan

${ }^{9}$ Kavli Institute for the Physics and Mathematics of the Universe (WPI), Todai Institutes for Advanced Study, University of Tokyo, Kashiwa, Chiba, Japan

${ }^{10}$ Information Engineering Graduate School of Science and Technology, Shinshu University, Nagano, Nagano, Japan

${ }^{11}$ Faculty of Engineering, Kanagawa University, Yokohama, Kanagawa, Japan

${ }^{12}$ Interdisciplinary Graduate School of Medicine and Engineering, University of Yamanashi, Kofu, Yamanashi, Japan

${ }^{13}$ Academic Assembly School of Science and Technology Institute of Engineering, Shinshu University, Nagano, Nagano, Japan

${ }^{14}$ Astrophysical Big Bang Laboratory, RIKEN, Wako, Saitama, Japan

${ }^{15}$ Department of Physics, Sungkyunkwan University, Jang-an-gu, Suwon, Korea

${ }^{16}$ Department of Physics, Tokyo City University, Setagaya-ku, Tokyo, Japan

${ }^{17}$ Institute for Nuclear Research of the Russian Academy of Sciences, Moscow, Russia

${ }^{18}$ Faculty of Systems Engineering and Science, Shibaura Institute of Technology, Minato-ku, Tokyo, Japan

${ }^{19}$ Department of Engineering Science, Faculty of Engineering, Osaka Electro-Communication University, Neyagawa-shi, Osaka, Japan

${ }^{20}$ Department of Physics, Chiba University, Chiba, Chiba, Japan

${ }^{21}$ Service de Physique Théorique, Université Libre de Bruxelles, Brussels, Belgium

${ }^{22}$ Department of Physics, Yonsei University, Seodaemun-gu, Seoul, Korea

${ }^{23}$ Center for Astrophysics and Cosmology, University of Nova Gorica, Nova Gorica, Slovenia

${ }^{24}$ Faculty of Science, Kochi University, Kochi, Kochi, Japan

${ }^{25}$ Nambu Yoichiro Institute of Theoretical and Experimental Physics, Osaka City University, Osaka, Osaka, Japan

${ }^{26}$ Department of Physical Sciences, Ritsumeikan University, Kusatsu, Shiga, Japan

${ }^{27}$ Quantum ICT Advanced Development Center, National Institute for Information and Communications Technology, Koganei, Tokyo, Japan

${ }^{28}$ Sternberg Astronomical Institute, Moscow M.V. Lomonosov State University, Moscow, Russia

${ }^{29}$ Department of Physics, School of Natural Sciences, Ulsan National Institute of Science and Technology, UNIST-gil, Ulsan, Korea

${ }^{30}$ Earthquake Research Institute, University of Tokyo, Bunkyo-ku, Tokyo, Japan

${ }^{31}$ Graduate School of Information Sciences, Hiroshima City University, Hiroshima, Hiroshima, Japan 
${ }^{32}$ Institute of Particle and Nuclear Studies, KEK, Tsukuba, Ibaraki, Japan

33 Graduate School of Science and Engineering, Tokyo Institute of Technology, Meguro, Tokyo, Japan

34 Department of Research Planning and Promotion, Quantum Medical Science Directorate, National Institutes for Quantum and Radiological Science and Technology, Chiba, Chiba, Japan

35 CEICO, Institute of Physics, Czech Academy of Sciences, Prague, Czech Republic

${ }^{36}$ Department of Physics and Institute for the Early Universe, Ewha Womans University, Seodaaemun-gu, Seoul, Korea

\section{Acknowledgements:}

The Telescope Array experiment is supported by the Japan Society for the Promotion of Science(JSPS) through Grants-in-Aid for Priority Area 431, for Specially Promoted Research JP21000002, for Scientific Research (S) JP19104006, for Specially Promoted Research JP15H05693, for Scientific Research (S) JP15H05741 and JP19H05607, for Science Research (A) JP18H03705, for Young Scientists (A) JPH26707011, and for Fostering Joint International Research (B) JP19KK0074, by the joint research program of the Institute for Cosmic Ray Research (ICRR), The University of Tokyo; by the Pioneering Program of RIKEN for the Evolution of Matter in the Universe (r-EMU); by the U.S. National Science Foundation awards PHY-1404495, PHY-1404502, PHY-1607727, PHY-1712517, PHY-1806797 and PHY-2012934; by the National Research Foundation of Korea (2017K1A4A3015188, 2020R1A2C1008230, \& 2020R1A2C2102800) ; by the Ministry of Science and Higher Education of the Russian Federation under the contract 075-15-2020778, RFBR grant 20-02-00625a (INR), IISN project No. 4.4501.18, and Belgian Science Policy under IUAP VII/37 (ULB). This work was partially supported by the grants ofThe joint research program of the Institute for Space-Earth Environmental Research, Nagoya University and Inter-University Research Program of the Institute for Cosmic Ray Research of University of Tokyo. The foundations of Dr. Ezekiel R. and Edna Wattis Dumke, Willard L. Eccles, and George S. and Dolores Doré Eccles all helped with generous donations. The State of Utah supported the project through its Economic Development Board, and the University of Utah through the Office of the Vice President for Research. The experimental site became available through the cooperation of the Utah School and Institutional Trust Lands Administration (SITLA), U.S. Bureau of Land Management (BLM), and the U.S. Air Force. We appreciate the assistance of the State of Utah and Fillmore offices of the BLM in crafting the Plan of Development for the site. Patrick A. Shea assisted the collaboration with valuable advice and supported the collaboration's efforts. The people and the officials of Millard County, Utah have been a source of steadfast and warm support for our work which we greatly appreciate. We are indebted to the Millard County Road Department for their efforts to maintain and clear the roads which get us to our sites. We gratefully acknowledge the contribution from the technical staffs of our home institutions. An allocation of computer time from the Center for High Performance Computing at the University of Utah is gratefully acknowledged.

\section{Yakutsk EAS Array Collaboration:}

E. A. Atlasov ${ }^{1}$, N. G. Bolotnikov ${ }^{1}$, N. A. Dyachkovskiy ${ }^{1}$, N. S. Gerasimova ${ }^{1}$, A. V. Glushkov ${ }^{1}$, A. A. Ivanov ${ }^{1}$, O. N. Ivanov ${ }^{1}$, I. A. Kellarev ${ }^{1}$, S. P. Knurenko ${ }^{1}$, A. D. Krasilnikov ${ }^{1}$, I. V. Ksenofontov ${ }^{1}$, L. T. Ksenofontov ${ }^{1}$, K. G. Lebedev ${ }^{1}$, S. V. Matarkin ${ }^{1}$, V. P. Mokhnachevskaya ${ }^{1}$, N. I. Neustroev ${ }^{1}$, I. S. Petrov ${ }^{1}$, A. S. Proshutinsky ${ }^{1}$, A. V. Sabourov ${ }^{1}$, I. Ye. Sleptsov ${ }^{1}$, G. G. Struchkov ${ }^{1}$, L. V. Timofeev ${ }^{1}$, B. B. Yakovlev ${ }^{1}$

${ }^{1}$ Yu. G. Shafer Institute of Cosmophysical Research and Aeronomy SB RAS 677980 Yakutsk, Russia

\section{Acknowledgements:}

The Yakutsk EAS Array is supported by the Ministry of Science and Higher Education of the Russian Federation under the contract AAAA-A21-121011990011-8. 